\title{
$s \ell(2)_{-4}$ WZW Model as an $N=4$-Supersymmetric Bosonic String WITH $c=-2$ MATTER
}

\author{
A. M. Semikhatov \\ and \\ I. Yu. Tipunin \\ I.E. Tamm Theory Division, P. N. Lebedev Physics Institute \\ Russian Academy of Sciences, 53 Leninski prosp., Moscow 117924, Russia
}

\begin{abstract}
We consider the $s \ell(2)$ current algebra at level $k=-4$ when the $s \ell(2)$ BRST operator is nilpotent. We formulate a spectral sequence converging to the cohomology of this BRST operator. At the second term of the spectral sequence, we observe an $N=4$ algebra. This algebra is generated in a $c=-2$ bosonic string whose BRST operator $\mathcal{Q}_{\text {string }}$ represents the next term in the spectral

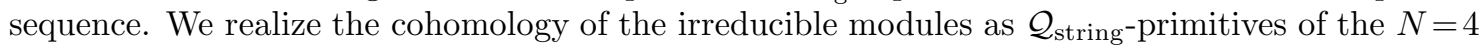
singular vectors and point out their relation to Lian-Zuckerman states of $c=-2$ matter. The relation between $s \ell(2)_{-4}$ WZW model and $c=-2$ bosonic string is established both at the level of BRST cohomology and at the level of an explicit operator construction. The relation of the $N=4$ algebra to the known symmetries of matter+gravity systems is also investigated.
\end{abstract}




\section{Introduction}

In this paper we will consider the $s \ell(2)$ current algebra at level $k=-4$. The BRST operator is then nilpotent quantum-mechanically without the introduction of an auxiliary set of currents, and the cohomology problem can be posed for the algebra itself (with the appropriate ghosts) rather than for a coset space. The cosets of $s \ell(2)$ for arbitrary $k$ have been considered in a number of papers, e.g. in 11, 2, 3, 团. Choosing $k=-4$ allows us to use several specific tools, such as a spectral sequence that converges to the $s \ell(2)$ BRST cohomology and leads to uncovering an underlying (level-1) $N=4$ superconformal algebra. The $s l(2)$ singular vectors then take the form of singular vectors in an $N=4$ module, and these can be used to produce Lian-Zuckerman states [6] in the corresponding $c=-2$-matter + gravity theory.

The correspondence between the $s \ell(2)$ WZW model and a matter+gravity theory, which follows from the cohomological analysis, can also be arrived at in rather explicit terms, by using a representation for $s \ell(2)$ currents obtained by inverting the hamiltonian reduction [7]. This representation is built by tensoring a Virasoro Verma module with free-field modules of the Liouville and ghost fields in such a way that the Hamiltonian reduction maps it back into the Virasoro module, while the Liouville and ghost fields correspond to those of a non-critical bosonic string. The matter theory is chosen according to the $s \ell(2)$ level $k$ and in our case of $k=-4$, the $s \ell(2)$ representation in question leads to an explicit relation between the $s \ell(2)_{k=-4}$ algebra and the bosonic string with $c=-2$ matter [8] (cf. the previous analysis [9] of the $k=-3$ WZW model which has lead to $c=1$ matter).

The mappings between $s \ell(2)$ and matter+gravity models can be viewed in a more general setting of Universal string theory [12, 13, 14, in which equivalences between theories with different underlying symmetry algebras are established. Two models of conformal field theories are usually considered equivalent if they lead to identical physical results, which can be taken to hold in (at least) three ways: as equivalence of their BRST cohomologies, as isomorphism of the correlators, or as 'reducibility' of singular vectors of one of the underlying algebras to those of the other algebra. These three approaches to establishing isomorphisms between different theories are heuristically equivalent. Indeed, correlators (to be precise, conformal blocks) in conformal field theories are solutions to differential equations derived from vanishing conditions of singular vectors. Singular vectors are in turn related to BRST cohomology in the following way. One observes that singular vectors in Verma modules are BRST-exact, $|\operatorname{sing}\rangle=\mathcal{Q}_{0}|\psi\rangle$, where $|\psi\rangle$ cannot be BRST-exact; now in the (usually irreducible) module obtained by factoring with respect to a submodule generated by $|\operatorname{sing}\rangle,|\psi\rangle$ becomes BRST-closed and therefore represents a state in the cohomology. Within the BRST-cohomology approach, a useful tool for establishing isomorphism between cohomology spaces is given by homotopy transformations. A homotopy transformation is required to provide a splitting of the BRST-operator of a given theory into a sum of two BRST-operators, one of which has trivial cohomology and the other coincides with the BRST operator of another theory, whose equivalence to the first theory is to be established. However, such an explicit mapping between the respective BRST cohomology spaces is not always easy to find; fortunately, the homotopy transformation approach is a special case of a more powerful technique of spectral sequences: a homotopy transformation can be viewed as such a lucky choice of grading that results in a spectral sequence with only two terms. In general, spectral sequences contain more terms and therefore have a broader applicability. On the other hand, they are less convenient, as compared with homotopy transformations, for establishing explicit mappings between states in the cohomology.

In this paper, we will trace, both at the cohomological level and in terms of explicit operator constructions, the relation between $s \ell(2)_{-4}$ theory and the $c=-2$ matter dressed with ghosts and 
Liouville fields. We will introduce a spectral sequence on the $s \ell(2)_{-4}$ BRST complex and use it in combination with an explicit operator realization of the $s \ell(2)$ currents. The $s \ell(2)$ representation that we use is not a free-field 'bosonization', as it involves as a building-block an arbitrary Virasoro Verma module which need not be bosonized through free fields. As a result, it does not lead to an 'accidental' vanishing of singular vectors and therefore can be applied to the description of the cohomology in irreducible modules 'generated' by singular vectors as explained above. We will thus systematically work with singular vectors rather than with the corresponding cohomology elements.

It is a remarkable fact that a generating construction [5] (to be referred to as MFF) is known for the $s \ell(2)$ (in fact, $s \ell(n)$ ) singular vectors. Our representation for $s \ell(2)$ currents can be made 'compatible' with the spectral sequence converging to the BRST cohomology, which will allow us to map the MFF singular vectors into a class of singular vectors in a representation of the $(N=4)_{k=1}$ algebra (the corresponding reformulation of the whole MFF generating formula would then be a variation on a similar construction for the $N=2$ algebra [30]). These $N=4$ singular vectors give rise to the $c=-2$ Lian-Zuckerman states, thereby providing a 'Lie-algebraic' origin of the latter. Their ghost numbers, in particular, can be derived from the embedding diagram of the $s \ell(2)_{k=-4}$ Verma modules. We will also consider relations of the $N=4$ algebra emerging in the spectral sequence with other algebras known to be relevant in non-critical bosonic strings 110, 11].

In section 2, we introduce a spectral sequence for the $s \ell(2)_{-4}$ BRST operator and observe an $N=4$ algebra in its first term. In section 3 , we map the $s \ell(2)_{-4}$ singular vectors into singular vectors in an $N=4$ module; then the $s \ell(2)_{-4}$ cohomology can be considered entirely in $N=4$ terms. We also give here the embedding diagram of the $s \ell(2)_{k=-4}$ Verma modules, which will then 'project' onto the $c=28$ Virasoro Verma module embedding diagram, related to the Lian-Zuckerman states. In section 4 , the use of the $s \ell(2)$ representation from ref. [7] leads us to identifying a $c=-2$ bosonic string 'inside' the $s \ell(2)_{-4}$ theory. The formula for $N=4$ singular vectors then projects, on the one hand, into singular vectors in the $c=28$ Verma module, and on the other hand, provides us with a construction for a class of Lian-Zuckerman states in the $c=-2$ bosonic string. In section 5 , we

finally discuss the relation of the observed $N=4$ symmetry with the known symmetries [10, 11] of matter dressed with gravity. The appearance of the $N=4$ algebra will be interpreted as a result of fitting together two twisted $N=2$ algebras known to exist when dressing a matter theory with ghosts and the Liouville 10.

\section{$2 \quad s \ell(2)$ BRST operator, spectral sequence, and $N=4$}

\section{$2.1 s \ell(2)$ currents, ghosts, and the BRST operator}

In this subsection, we fix our notations and introduce the BRST complex, Weyl group action, and the representations of $s \ell(2)$ we are going to consider in this paper. To begin with, the $s \ell(2)$ current algebra operator products are taken in the form

$$
\begin{aligned}
J^{0}(z) J^{ \pm}(w) & = \pm \frac{J^{ \pm}}{z-w} \\
J^{+}(z) J^{-}(w) & =\frac{-k / 2}{(z-w)^{2}}-\frac{J^{0}}{z-w} \\
J^{0}(z) J^{0}(w) & =\frac{k / 2}{(z-w)^{2}}
\end{aligned}
$$


We also introduce three ghost systems $\mathcal{B}^{+}, \mathcal{C}_{+} ; \mathcal{B}^{0}, \mathcal{C}_{0}$, and $\mathcal{B}^{-}, \mathcal{C}_{-}$associated with the $\operatorname{s\ell }(2)$ generators $J^{+}, J^{0}$ and $J^{-}$respectively. Together, these make up an algebra

$$
\mathcal{A}=s \ell(2) \oplus\left[\mathcal{B}^{+}, \mathcal{C}_{+}\right] \oplus\left[\mathcal{B}^{-}, \mathcal{C}_{-}\right] \oplus\left[\mathcal{B}^{0}, \mathcal{C}_{0}\right]
$$

where $\left[\mathcal{B}^{+}, \mathcal{C}_{+}\right],\left[\mathcal{B}^{-}, \mathcal{C}_{-}\right]$and $\left[\mathcal{B}^{0}, \mathcal{C}_{0}\right]$ are superalgebras spanned by the corresponding ghost systems. The full energy-momentum tensor, which will be denoted by $\mathcal{T}_{\mathcal{A}}$, is equal to the sum of Sugawara and ghost energy-momentum tensors and for $k=-4$ reads

$$
\mathcal{T}_{\mathcal{A}}=\frac{1}{2}\left(-J^{0} J^{0}+J^{+} J^{-}+J^{-} J^{+}\right)-\mathcal{B}^{+} \partial \mathcal{C}_{+}-\mathcal{B}^{-} \partial \mathcal{C}_{-}-\mathcal{B}^{0} \partial \mathcal{C}_{0}
$$

All the $\mathcal{B}$ ghosts thus have dimension 1. In the formula (2.3) and other similar equations below, the ghost monomials are normal-ordered with respect to the $s l_{2}$-invariant ghosts vacua.

For $k=-4$, the algebra $\mathcal{A}$ is made into a BRST complex by introducing the BRST current according to the standard recipe [15]

$$
\mathcal{J}_{\mathcal{A}}=\mathcal{C}_{+} J^{+}+\mathcal{C}_{0} J^{0}+\mathcal{C}_{-} J^{-}-\mathcal{C}_{-} \mathcal{C}_{0} \mathcal{B}^{-}-\mathcal{C}_{0} \mathcal{C}_{+} \mathcal{B}^{+}-\mathcal{C}_{-} \mathcal{C}_{+} \mathcal{B}^{0}
$$

The corresponding BRST charge

$$
\mathcal{Q}_{\mathcal{A}}=\oint \mathcal{J}_{\mathcal{A}}
$$

is indeed nilpotent, $\mathcal{Q}_{\mathcal{A}}^{2}=0$, when $k$ is equal to minus two Coxeter numbers, $k=-4$ [1] [. In this case the BRST current is also OPE-isotropic, $\mathcal{J}_{\mathcal{A}}(z) \mathcal{J}_{\mathcal{A}}(w)=0$. In the following, $k$ will be set equal to -4 .

The energy-momentum tensor (2.3) turns out to be BRST-exact:

$$
\mathcal{T}_{\mathcal{A}}(z)=\left[\mathcal{Q}_{\mathcal{A}}, \mathcal{G}_{\mathcal{A}}(z)\right], \quad \mathcal{G}_{\mathcal{A}}=\mathcal{B}^{+} J^{-}-\mathcal{B}^{0} J^{0}+\mathcal{B}^{-} J^{+}
$$

It follows then that all the states in the cohomology of $\mathcal{Q}_{\mathcal{A}}$ must have vanishing dimension. Another condition on the cohomology follows by considering the currents

$$
\widehat{J}^{ \pm, 0}=\left[\mathcal{Q}_{\mathcal{A}}, B^{ \pm, 0}\right], \quad\left\{\begin{aligned}
\widehat{J}^{+} & =J^{+}-\mathcal{B}^{+} \mathcal{C}_{0}+\mathcal{C}_{-} \mathcal{B}^{0} \\
\widehat{J}^{0} & =J^{0}+\mathcal{B}^{+} \mathcal{C}_{+}-\mathcal{B}^{-} \mathcal{C}_{-} \\
\widehat{J}^{-} & =J^{-}+\mathcal{B}^{-} \mathcal{C}_{0}-\mathcal{C}_{+} \mathcal{B}^{0}
\end{aligned}\right.
$$

that satisfy an $s \ell(2)$ algebra at level $k+4=0$ (hence, in particular, $\widehat{J}^{0}$ is OPE-isotropic). Since $\widehat{J}^{0}$ is BRST-trivial, states in the cohomology must have $\widehat{J}^{0}$-spin equal to zero.

The current $\mathcal{J}_{\mathcal{A}}$ itself is also BRST-exact,

$$
\mathcal{J}_{\mathcal{A}}=\left[\mathcal{Q}_{\mathcal{A}}, \mathcal{H}_{\mathcal{A}}\right], \quad \mathcal{H}_{\mathcal{A}} \equiv \mathcal{B}^{+} \mathcal{C}_{+}+\mathcal{B}^{-} \mathcal{C}_{-}+\mathcal{B}^{0} \mathcal{C}_{0}
$$

Note that the cohomology is naturally graded by zero mode of $\mathcal{H}_{\mathcal{A}}$. As is well known [1], cohomology of $\mathcal{Q}_{\mathcal{A}}$ is given by $H_{\text {rel }}^{*} \oplus\left(\mathcal{C}_{0}\right)_{0} H_{\text {rel }}^{*}$ where $H_{\text {rel }}^{*}$ denotes the relative cohomology, which will be the only one we are going to consider.

\footnotetext{
${ }^{1}$ To avoid misunderstanding, let us remind the reader that, in our normalization (which differs from that adopted in refs. 1. 16]), 'the other' critical value, at which the universal enveloping algebra acquires an infinite-dimensional center [17], is $k=-2$.
} 
It will be useful to extend the action of the affine Weyl group $\tilde{W}$ on the $\mathcal{B}^{ \pm} \mathcal{C}_{ \pm}$ghosts by demanding that $\tilde{W}$ act on the $\widehat{J}$ currents in the same way as it acts on $J^{ \pm, 0}$, namely, for $\tilde{W} \ni(s, \ell)$ where $s \in W$ is + or - and $\ell$ is an element of the weight lattice,

$$
\begin{gathered}
(s, \ell) . \widehat{J}_{n}^{\alpha}=\widehat{J}_{n+\alpha \ell}^{s \alpha}, \\
(s, \ell) . \widehat{J}_{n}^{0}=s \widehat{J}_{n}^{0} .
\end{gathered}
$$

It follows then that

$$
\begin{aligned}
(s, \ell) \cdot \mathcal{B}_{n}^{\alpha} & =s \mathcal{B}_{n+\alpha \ell}^{s \alpha}, \\
(s, \ell) \cdot\left(\mathcal{C}_{\alpha}\right)_{n} & =s\left(\mathcal{C}_{s \alpha}\right)_{n-\alpha \ell} .
\end{aligned}
$$

Now we specify a representation of the algebra (2.2). Consider a highest-weight representation of $s \ell(2)$ with an integral spin $j$ :

$$
\begin{aligned}
J_{0}^{0}|j\rangle & =j|j\rangle, \quad J_{n}^{0}|j\rangle=0, \quad n \geq 1 \\
J_{n}^{+}|j\rangle & =0, \quad n \geq 0 \\
J_{n}^{-}|j\rangle & =0, \quad n \geq 1
\end{aligned}
$$

and tensor it with the corresponding ghost vacua into a 'highest-weight' of $\mathcal{A}$ :

$$
|j\rangle_{\mathcal{A}}= \begin{cases}|j\rangle \otimes|j+1\rangle_{+} \otimes|0\rangle_{0} \otimes|1\rangle_{-} & j \geq 0, \\ |j\rangle \otimes|0\rangle_{+} \otimes|0\rangle_{0} \otimes|-j\rangle_{-} & j<0\end{cases}
$$

The ghost vacua $|0\rangle_{ \pm, 0}$ are defined as follows. For a $b c$ system of dimension $\lambda$, we define $|q\rangle$ by 18

$$
b_{\geq 1-\lambda+q}|q\rangle=0, \quad c_{\geq \lambda-q}|q\rangle=0
$$

(this is $s l_{2}$-invariant for $q=0$ ). This state has dimension

$$
\Delta_{\lambda}(q)=\frac{1}{2} q(q+1-2 \lambda),
$$

the formula to be used when dressing $s \ell(2)$ states with ghosts so as to get dimension-0 states (as will be necessary for states in the cohomology). All monomials in $b, c$ will always be assumed normal-ordered with respect to the $s l_{2}$-invariant vacuum $|0\rangle$. Then, in particular

$$
(b c)_{0}|q\rangle=-q|q\rangle \text {, }
$$

which explains the choice of ghost states in (2.12), yielding the vanishing $\widehat{J}^{0}$-spin. Now, to return to (2.13), the ghost vacua in the formula (2.12) for $j>0$, for instance, are such that

$$
\begin{aligned}
\left(\mathcal{C}_{+}\right)_{n}|j+1\rangle_{+} & =0, & n \geq-j, & \mathcal{B}^{+}{ }_{n}|j+1\rangle_{+} & =0, & n \geq 1+j, \\
\left(\mathcal{C}_{0}\right)_{n}|0\rangle_{0} & =0, & n \geq 1, & \mathcal{B}^{0}{ }_{n}|0\rangle_{0} & =0, & n \geq 0 \\
\left(\mathcal{C}_{-}\right)_{n}|1\rangle_{-} & =0, & n \geq 0, & \mathcal{B}^{-}{ }_{n}|1\rangle_{-} & =0, & n \geq 1
\end{aligned}
$$

(recall that our ghost systems have dimensions $\lambda=1$ ).

The representation of the $\mathcal{A}$ algebra is built over the vacuum (2.12) by acting on it with the creation operators.

The $\widehat{J}^{0}$-spin-zero vacuum state $(2.12)$ can be considered as a representative of the only state in the cohomology of the Verma module for integral $j$. Simple considerations based on the 'compensation' of the $s \ell(2)$ spin $j$ by the ghost contributions show that there is not even a vacuum in the cohomology of the Verma module for half-integral $j$, and so we restrict ourselves to the case of integral $j$. In what follows, we will look for the cohomology of the irreducible modules obtained as factors of the Verma module. 


\subsection{Spectral sequence and $N=4$ algebra}

Since the energy-momentum tensor and the BRST current $\mathcal{J}_{\mathcal{A}}$ are BRST-exact, one might expect an underlying topological algebra in the system. This is indeed the case, and the topological algebra turns out to be a certain extension [16, 19] of the twisted $N=2$ algebra [20, 21]. It differs from the twisted $N=2$ by new terms in the operator product $\mathcal{G}_{\mathcal{A}} \cdot \mathcal{G}_{\mathcal{A}}$ (where $\mathcal{G}_{\mathcal{A}}$ is the superpartner to the energy-momentum tensor from eq. (2.6)):

$$
\mathcal{G}_{\mathcal{A}}(z) \mathcal{G}_{\mathcal{A}}(w)=\frac{\mathcal{W}}{z-w} \quad \text { where } \quad \mathcal{W}(z)=\left[\mathcal{Q}_{\mathcal{A}}, \mathcal{V}(z)\right] \quad \text { and } \quad \mathcal{V}=\mathcal{B}^{-} \mathcal{B}^{+} \mathcal{B}^{0}
$$

$\mathcal{V}$ and $\mathcal{W}$ generate a commutative ideal in the extended topological algebra, and the twisted $N=2$ algebra is a factor with respect to this ideal. This extended topological algebra is not very useful to work with (at least as compared to the true $N=2$ ), however, as we are going to show, this is not needed, since the first step in evaluating the cohomology of $\mathcal{Q}_{\mathcal{A}}$ will effectively lead to factoring over the ideal generated by $\mathcal{V}$ and $\mathcal{W}$.

Observe that a filtration $F^{i} \mathcal{A}$ exists on the BRST complex $\left(\mathcal{A}, \mathcal{Q}_{\mathcal{A}}\right)$ (i.e., the filtration is compatible with the action of the BRST operator in the sense that $\left.\mathcal{Q}_{\mathcal{A}}\left(F^{i} \mathcal{A}\right) \subset F^{i} \mathcal{A}\right)$. The filtration can be described by first assigning the following gradings to our fields:

$$
\begin{aligned}
& \operatorname{deg} \mathcal{C}_{0}=\operatorname{deg} \mathcal{B}^{0}=\operatorname{deg} J^{0}=\operatorname{deg} \mathcal{B}^{-}=\operatorname{deg} \mathcal{C}_{-}=0, \\
& \operatorname{deg} J^{-}=-\operatorname{deg} J^{+}=1, \\
& -\operatorname{deg} \mathcal{B}^{+}=\operatorname{deg} \mathcal{C}_{+}=3 .
\end{aligned}
$$

The algebra $\mathcal{A}$ is then decomposed into a direct sum of subspaces $G_{l}$ with definite degrees, and the filtration $\ldots \subset F^{i} \mathcal{A} \subset F^{i+1} \mathcal{A} \subset F^{i+2} \mathcal{A} \subset \ldots$ is defined by $F^{i} \mathcal{A}=\bigoplus_{l \geq i} G_{l}$. Then we can split the BRST current into a finite sum of terms with non-negative degrees:

$$
\mathcal{J}=\ldots+0+\mathcal{J}^{(0)}+\mathcal{J}^{(1)}+\mathcal{J}^{(2)}+\mathcal{J}^{(3)}+0+\ldots
$$

where

$$
\begin{aligned}
\mathcal{J}^{(0)} & =\mathcal{C}_{0} \widehat{J}^{0}, \\
\mathcal{J}^{(1)} & =\mathcal{C}_{-} J^{-}, \\
\mathcal{J}^{(2)} & =\mathcal{C}_{+} J^{+}, \\
\mathcal{J}^{(3)} & =\mathcal{C}_{+} \mathcal{C}_{-} \mathcal{B}^{0} .
\end{aligned}
$$

Since the degrees of all the non-vanishing terms in (2.19) are non-negative, there exists a spectral sequence associated with this filtration which converges to the cohomology of $\mathcal{Q}_{\mathcal{A}}$. Observe that the differentials $\mathcal{Q}^{(0)}, \mathcal{Q}^{(1)}, \mathcal{Q}^{(2)}, \mathcal{Q}^{(3)}$ corresponding to $\mathcal{J}^{(0)}, \mathcal{J}^{(1)}, \mathcal{J}^{(2)} \mathcal{J}^{(3)}$ respectively are nilpotent separately and $\mathcal{Q}^{(0)}$ anticommutes with $\mathcal{Q}^{(1)}$ and $\mathcal{Q}^{(2)}$. As can be seen from the form of $\mathcal{Q}^{(0)}=$ $\oint \mathcal{J}^{(0)}$, it effectively imposes the constraint $\widehat{J}^{0} \sim 0$. Further, $\mathcal{Q}^{(3)}$ is zero on the cohomology of $\mathcal{Q}^{(0)}$, hence the spectral sequence degenerates after the third term. Cohomology of the BRST operator (2.4) is therefore given by the cohomology of $\mathcal{Q}^{(2)}$ evaluated on the cohomology of $\mathcal{Q}^{(1)}$ which is evaluated on the cohomology of $\mathcal{Q}^{(0)}$.

As follows from a careful reading of the last phrase, the first step in the analysis of the spectral sequence consists therefore in restricting to the cohomology of $\mathcal{Q}^{(0)}$. This will have an immediate effect on the extended topological algebra referred to in the beginning of this subsection. Namely, 
the fields $\mathcal{V}$ and $\mathcal{W}$ vanish on the cohomology of $\mathcal{Q}^{(0)}$ since they consist of terms which are either $\mathcal{Q}^{(0)}$-exact or not $\mathcal{Q}^{(0)}$-closed. As a result, the extended topological algebra reduces to the twisted $N=2$ algebra [20, 21]. A useful choice of representatives of the $N=2$ algebra generators is given by

$$
\begin{aligned}
\widehat{\mathcal{J}}_{\mathcal{A}} & =\mathcal{C}_{-} J^{-}+\mathcal{C}_{+} J^{+}, \\
\widehat{\mathcal{G}}_{\mathcal{A}} & =\mathcal{B}^{-} J^{+}+\mathcal{B}^{+} J^{-}, \\
\widehat{\mathcal{T}}_{\mathcal{A}} & =J^{-} J^{+}+\partial \mathcal{B}^{+} \mathcal{C}_{+}-2 \mathcal{B}^{-} \partial \mathcal{C}_{-}-\partial \mathcal{B}^{-} \mathcal{C}_{-}+\frac{1}{2}\left(J^{0} \mathcal{B}^{+} \mathcal{C}_{+}-J^{0} \mathcal{B}^{-} \mathcal{C}_{-}+\partial J^{0}\right), \\
\widehat{\mathcal{H}}_{\mathcal{A}} & =\mathcal{B}^{+} \mathcal{C}_{+}+\mathcal{B}^{-} \mathcal{C}_{-} .
\end{aligned}
$$

These close to an $N=2$ algebra modulo $\mathcal{Q}^{(0)}$-exact terms.

The structure of the cohomology of $\mathcal{Q}^{(0)}$ can in fact be refined considerably by noticing that it bears a representation of an $N=4$ algebra that extends the above $N=2$ algebra. Representatives of the $N=4$ generators can be chosen as

$$
\begin{array}{rlrl}
\mathcal{T} & =\widehat{\mathcal{T}}_{\mathcal{A}}, & \mathcal{G}^{1} & =\mathcal{C}_{+} J^{+}, \\
J_{N=4}^{+} & =\mathcal{C}_{-} \mathcal{C}_{+}, & \mathcal{G}^{2} & =\mathcal{B}^{-} J^{+}, \\
J_{N=4}^{0} & =-\frac{1}{2} \widehat{\mathcal{H}}_{\mathcal{A}}, & \overline{\mathcal{G}}_{1}=\mathcal{B}^{+} J^{-}, \\
J_{N=4}^{-}=\mathcal{B}^{-} \mathcal{B}^{+}, & \overline{\mathcal{G}}_{2}=\mathcal{C}_{-} J^{-} .
\end{array}
$$

Then it can be checked that the following $N=4$ OPEs [22] are satisfied modulo $\mathcal{Q}^{(0)}$-exact terms ${ }^{2}$ :

$$
\begin{aligned}
& J_{N=4}^{\alpha}(z) \mathcal{G}^{a}(w)=-\frac{\left(\sigma^{\alpha}\right)_{b}^{a} \mathcal{G}^{b}}{z-w}, \quad J_{N=4}^{\alpha}(z) \overline{\mathcal{G}}_{a}(w)=\frac{\overline{\mathcal{G}}_{b}\left(\sigma^{\alpha}\right)_{a}^{b}}{z-w}, \\
& \mathcal{T}(z) \mathcal{G}^{a}(w)=\frac{a \mathcal{G}^{a}(w)}{(z-w)^{2}}+\frac{\partial \mathcal{G}^{a}}{z-w}, \quad \mathcal{T}(z) \overline{\mathcal{G}}_{a}(w)=\frac{(3-a) \overline{\mathcal{G}}_{a}(w)}{(z-w)^{2}}+\frac{\partial \overline{\mathcal{G}}_{a}}{z-w} \\
& \mathcal{T}(z) J_{N=4}^{\alpha}(w)=\frac{-\delta_{\alpha, 0}}{(z-w)^{3}}+\frac{(1-\alpha) J_{N=4}^{\alpha}(w)}{(z-w)^{2}}+\frac{\partial J_{N=4}^{\alpha}}{z-w}, \quad \alpha=+1,0,-1, \\
& \mathcal{G}^{a}(z) \mathcal{G}^{b}(w)=0, \quad \overline{\mathcal{G}}_{a}(z) \overline{\mathcal{G}}_{b}(w)=0, \\
& \mathcal{G}^{a}(z) \overline{\mathcal{G}}_{b}(w)=\frac{2 \delta_{b}^{a}}{(z-w)^{3}}-\frac{2\left(\sigma^{\alpha}\right)_{b}^{a} \eta_{\alpha \beta} J_{N=4}^{\beta}(w)}{(z-w)^{2}}+\frac{-\left(\sigma^{\alpha}\right)_{b}^{a} \eta_{\alpha \beta} \partial J_{N=4}^{\beta}+\delta_{b}^{a}\left(\mathcal{T}-\partial J_{N=4}^{0}\right)}{z-w}
\end{aligned}
$$

where $J_{N=4}^{+}, J_{N=4}^{0}$ and $J_{N=4}^{-}$make up an $s \ell(2)$ algebra at level 1:

$$
\begin{aligned}
& J_{N=4}^{0}(z) J_{N=4}^{ \pm}(w)=\frac{J_{N=4}^{ \pm}}{z-w}, \quad J_{N=4}^{+}(z) J_{N=4}^{-}(w)=-\frac{1}{(z-w)^{2}}-\frac{2 J_{N=4}^{0}}{z-w}, \\
& J_{N=4}^{0}(z) J_{N=4}^{0}(w)=\frac{1 / 2}{(z-w)^{2}} .
\end{aligned}
$$

${ }^{2}$ Greek superscripts and subscripts $\alpha=0, \pm$ denote $s \ell(2)$ triplets, while Latin superscripts and subscripts $a, b$ run over 1,2 and label $s \ell(2)$ doublet and antidoublet representations. The sigma matrices $\sigma^{0}, \sigma^{+}$and $\sigma^{-}$are defined as:

$$
\sigma^{0}=\left(\begin{array}{cc}
-\frac{1}{2} & 0 \\
0 & \frac{1}{2}
\end{array}\right) \quad \sigma^{+}=\left(\begin{array}{cc}
0 & 0 \\
1 & 0
\end{array}\right) \quad \sigma^{-}=\left(\begin{array}{cc}
0 & -1 \\
0 & 0
\end{array}\right)
$$

Superscripts label rows and subscripts, columns. The metric tensor $\eta_{\alpha \beta}$ is: $\frac{1}{2} \eta_{00}=-\eta_{+-}=-\eta_{-+}=1$ and other components are equal to zero. 
This is in fact a twisted algebra, in particular $J_{N=4}^{ \pm}$have dimensions $1 \mp 1$, so that the corresponding commutation relations read:

$$
\left[\left(J_{N=4}^{+}\right)_{m},\left(J_{N=4}^{-}\right)_{n}\right]=-\delta_{m+n, 0}(m-1)-2\left(J_{N=4}^{0}\right)_{m+n}
$$

The Weyl group action (2.9), (2.10) carries over to the $N=4$ algebra. Translations along the weights from the affine Weyl group act on the generators trivially, while the reflection '-' acts as

$$
\begin{aligned}
\mathcal{T} & \mapsto \mathcal{T}, & \mathcal{G}^{1} & \mapsto-\overline{\mathcal{G}}_{2}, \\
J_{N=4}^{+} & \mapsto-J_{N=4}^{+}, & \mathcal{G}^{2} & \mapsto-\overline{\mathcal{G}}_{1}, \\
J_{N=4}^{0} & \mapsto J_{N=4}^{0}, & \overline{\mathcal{G}}_{1} & \mapsto-\mathcal{G}^{2}, \\
J_{N=4}^{-} & \mapsto-J_{N=4}^{-}, & \overline{\mathcal{G}}_{2} & \mapsto-\mathcal{G}^{1}
\end{aligned}
$$

where the minus signs in front of the fermionic generators can be omitted without affecting the $N=4$ commutation relations. Evaluating the transformation of $\mathcal{T}$ when this $N=4$ generator is represented as in (2.22), we find, literally, $\mathcal{T} \mapsto \mathcal{T}+2 \partial \widehat{J}^{0}$ but this does not actually change $\mathcal{T}$ as an element in the cohomology of $\mathcal{Q}^{(0)}$.

Note also that $N=4$ algebra admits, along with (2.26), another automorphism:

$$
\begin{array}{rll}
\mathcal{T} & \rightarrow \mathcal{T}-2 \partial J_{N=4}^{0}, & \mathcal{G}^{1} \rightarrow \mathcal{G}^{2}, \\
J_{N=4}^{+} \rightarrow-J_{N=4}^{-}, & \mathcal{G}^{2} \rightarrow \mathcal{G}^{1} \\
J_{N=4}^{0} \rightarrow-J_{N=4}^{0}, & \overline{\mathcal{G}}_{1} \rightarrow \overline{\mathcal{G}}_{2} \\
J_{N=4}^{-} \rightarrow-J_{N=4}^{+}, & \overline{\mathcal{G}}_{2} \rightarrow \overline{\mathcal{G}}_{1}
\end{array}
$$

which is induced in the construction (2.22) by interchanging the ghosts as

$$
\begin{array}{llll}
\mathcal{B}^{+} & \rightarrow \mathcal{C}_{-}, & \mathcal{C}_{-} & \rightarrow \mathcal{B}^{+} \\
\mathcal{C}_{+} & \rightarrow \mathcal{B}^{-}, & \mathcal{B}^{-} & \rightarrow \mathcal{C}_{+} .
\end{array}
$$

\subsection{Representations}

Now let us see what representation of the $N=4$ algebra is arrived at starting with an $s \ell(2)$ representation. We consider the $s \ell(2)$ highest-weight representations built on highest weights $|j\rangle$ with $j=j_{+}(r, s)$ or $j_{-}(r, s)$, labeled by two positive integers $r$ and $s$ via

$$
j_{+}(r, s)=\frac{1}{2}(r-1)-\frac{1}{2}(k+2)(s-1), \quad r, s \geq 1
$$

and

$$
j_{-}(r, s)=-\frac{1}{2}(r+1)+\frac{1}{2}(k+2) s, \quad r, s \geq 1 .
$$

Such states will be eigenstates of the $N=4$ current $J_{N=4}^{0}$ : we evaluate $2\left(J_{N=4}^{0}\right)_{0}$ on $|j\rangle_{\mathcal{A}}$ as

$$
2\left(J_{N=4}^{0}\right)_{0}|j\rangle_{\mathcal{A}}= \begin{cases}(j+2)|j\rangle_{\mathcal{A}}, & j+2=\frac{r-1}{2}+s+1 \geq 2, \\ -j|j\rangle_{\mathcal{A}}, & -j=\frac{r+1}{2}+s \geq 2\end{cases}
$$


Therefore, fixing a $\mathrm{j} \geq 2$ that will be the eigenvalue of $2\left(J_{N=4}^{0}\right)_{0}-($ twice $)$ the $N=4$ spin - we arrive at two $N=4$ states defined in the following way: for each of these states,

$$
\begin{aligned}
\mathcal{L}_{n}|\mathrm{j}, \pm\rangle_{N=4} & =0, \quad n \geq 0, & & \\
\left(J_{N=4}^{0}\right)_{n}|\mathrm{j}, \pm\rangle_{N=4} & =0, \quad n \geq 1, & & \left(J_{N=4}^{+}\right)_{n}|\mathrm{j}, \pm\rangle_{N=4}=0, \quad n \geq-\mathrm{j}+1, \\
2\left(J_{N=4}^{0}\right)_{0}|\mathrm{j}, \pm\rangle_{N=4} & =\mathrm{j}|\mathrm{j}, \pm\rangle_{N=4}, & & \left(J_{N=4}^{-}\right)_{n}|\mathrm{j}, \pm\rangle_{N=4}=0, \quad n \geq \mathrm{j}-1,
\end{aligned}
$$

while

$$
\begin{array}{ll}
\left(\mathcal{G}^{1}\right)_{n}|\mathrm{j},+\rangle_{N=4}=0, \quad n \geq-\mathrm{j}+1, & \left(\mathcal{G}^{2}\right)_{n}|\mathrm{j},+\rangle_{N=4}=0, \quad n \geq 0, \\
\left(\overline{\mathcal{G}}_{1}\right)_{n}|\mathrm{j},+\rangle_{N=4}=0, \quad n \geq \mathrm{j}-1, & \left(\overline{\mathcal{G}}_{2}\right)_{n}|\mathrm{j},+\rangle_{N=4}=0, \quad n \geq 0
\end{array}
$$

whereas

$$
\begin{array}{ll}
\left(\mathcal{G}^{1}\right)_{n}|\mathrm{j},-\rangle_{N=4}=0, \quad n \geq 0, & \left(\mathcal{G}^{2}\right)_{n}|\mathrm{j},-\rangle_{N=4}=0, n \geq \mathrm{j}-1, \\
\left(\overline{\mathcal{G}}_{1}\right)_{n}|\mathrm{j},-\rangle_{N=4}=0, \quad n \geq 0, & \left(\overline{\mathcal{G}}_{2}\right)_{n}|\mathrm{j},-\rangle_{N=4}=0, n \geq-\mathrm{j}+1 .
\end{array}
$$

Note that the two types of highest-weight conditions, eqs. (2.33) and (2.34), are related by the Weyl reflection (2.26) on the $N=4$ generators $[$.

One can notice that the above 'skewed' highest-weight states $|\mathrm{j}, \pm\rangle_{N=4}$ are related to $N=4$ highest weights that exist in the series of spin- $\frac{1}{2}(N=4)_{k=1}$ representations labeled by conformal dimensions [23]. Namely, consider a highest-weight state $\left|\frac{1}{2}, \Delta\right\rangle_{N=4}$ satisfying the conditions

$$
\begin{aligned}
& \mathcal{L}_{\geq 1}\left|\frac{1}{2}, \Delta\right\rangle_{N=4}=\left(J_{N=4}^{ \pm, 0}\right)_{\geq 1}\left|\frac{1}{2}, \Delta\right\rangle_{N=4}=\left(\mathcal{G}^{a}\right)_{\geq 1}\left|\frac{1}{2}, \Delta\right\rangle_{N=4}=\left(\overline{\mathcal{G}}_{a}\right)_{\geq 1}\left|\frac{1}{2}, \Delta\right\rangle_{N=4}=0, \\
& \left(\mathcal{G}^{2}\right)_{0}\left|\frac{1}{2}, \Delta\right\rangle_{N=4}=\left(\overline{\mathcal{G}}_{1}\right)_{0}\left|\frac{1}{2}, \Delta\right\rangle_{N=4}=0, \\
& \mathcal{L}_{0}\left|\frac{1}{2}, \Delta\right\rangle_{N=4}=\Delta\left|\frac{1}{2}, \Delta\right\rangle_{N=4}, \quad\left(J_{N=4}^{0}\right)_{0}\left|\frac{1}{2}, \Delta\right\rangle_{N=4}=\frac{1}{2}\left|\frac{1}{2}, \Delta\right\rangle_{N=4} .
\end{aligned}
$$

(all the Verma modules built over any $\left|\frac{1}{2}, \Delta\right\rangle_{N=4}$ except $\left|\frac{1}{2}, 0\right\rangle_{N=4}$ are not unitary; The $\left|\frac{1}{2}, 0\right\rangle_{N=4}$ representation is called massless [23]).

In ref. [23], singular vectors of $N=4$ algebra were arrived at by restricting to a representation of the $s \ell(2)$ subalgebra of the $N=4$ algebra and noticing that singular vectors of the $s \ell(2)$ subalgebra are singular vectors of $N=4$ algebra. For unitary representations, other singular vectors are absent [23]. As we will see, more singular vectors exist for non-unitary representations. In our case we have a realization of the $N=4$ algebra in which the $s \ell(2)$ subalgebra is constructed out of two ghost pairs, and therefore all singular vectors of ref. [23] vanish. Thus the $N=4$ representations 'induced' from the $s \ell(2)_{-4}$ Verma module tensored with ghosts are related to $N=4$ Verma modules after factorization of the latter with respect to singular vectors from ref. [23]: namely, the 'skewed' highest-weight states characterized by (2.32)-(2.34), and the highest-weights (2.35) are related via

$$
|\mathrm{j},+\rangle=\left(\mathcal{G}^{1}\right)_{-\mathrm{j}+1} \cdots\left(\mathcal{G}^{1}\right)_{-1}\left|\frac{1}{2}, \Delta\right\rangle_{N=4} \quad \text { when } \Delta=-\frac{1}{2} \mathrm{j}(\mathrm{j}-1)
$$

and, similarly,

$$
\left|\frac{1}{2}, \Delta\right\rangle_{N=4}=\left(\mathcal{G}^{2}\right)_{0} \ldots\left(\mathcal{G}^{2}\right)_{\mathrm{j}-2}|\mathrm{j},-\rangle \quad \text { when } \Delta=-\frac{1}{2} \mathrm{j}(\mathrm{j}-3)-1
$$

\footnotetext{
${ }^{3}$ Note similar 'skewed' highest-weight conditions in 24].
} 
Heuristically, our states $|\mathbf{j}, \pm\rangle$ are each a 'half' of the simplest singular (or co-singular) vector in the 'proper' highest-weight module. The factorization leads to fulfillment of several conditions, including

$$
\begin{array}{lll}
\left(\left(J_{N=4}^{-}\right)_{0}\right)^{2}\left|\frac{1}{2}, \Delta\right\rangle_{N=4}=0, & \left(\mathcal{G}^{2}\right)_{0}\left(J_{N=4}^{-}\right)_{0}\left|\frac{1}{2}, \Delta\right\rangle_{N=4}=0, & \left(\overline{\mathcal{G}}_{1}\right)_{0}\left(J_{N=4}^{-}\right)_{0}\left|\frac{1}{2}, \Delta\right\rangle_{N=4}=0, \\
\left(\left(J_{N=4}^{+}\right)_{0}\right)^{2}\left|\frac{1}{2}, \Delta\right\rangle_{N=4}=0, & \left(\mathcal{G}^{1}\right)_{0}\left(J_{N=4}^{+}\right)_{0}\left|\frac{1}{2}, \Delta\right\rangle_{N=4}=0, & \left(\overline{\mathcal{G}}_{2}\right)_{0}\left(J_{N=4}^{+}\right)_{0}\left|\frac{1}{2}, \Delta\right\rangle_{N=4}=0 .
\end{array}
$$

These vanishing conditions will be used in the next section.

The states $\left|\frac{1}{2}, \Delta\right\rangle_{N=4}$ (except $\left|\frac{1}{2}, 0\right\rangle_{N=4}=|-1\rangle_{\mathcal{A}}$ ) cannot be represented in intrinsic terms of the algebra $\mathcal{A}$, i.e. in terms of the $s \ell(2)_{-4}$ algebra and ghosts. Constructing the $\left|\frac{1}{2}, \Delta\right\rangle_{N=4}$ states requires identifying in the $s \ell(2)$ representation a matter sector (the result of Hamiltonian reduction) and the 'complementary' ghost sectors, as will be shown in section 4 .

To return to the spectral sequence, observe now that the differentials $\mathcal{Q}^{(1)}$ and $\mathcal{Q}^{(2)}$ from 2.20 ) are given by zero modes of the $N=4$ generators $\overline{\mathcal{G}}_{2}$ and $\mathcal{G}^{1}$ respectively. Therefore the cohomology of $\mathcal{Q}_{\mathcal{A}}(2.5)$ will be given in the $N=4$ terms as the cohomology of $\left(\mathcal{G}^{1}\right)_{0}$ evaluated on the cohomology of $\left(\overline{\mathcal{G}}_{2}\right)_{0}$. Recall further that, while there is only a vacuum in the cohomology of the Verma module, factoring with respect to submodules generated by singular vectors gives rise to non-trivial cohomology; the standard 'cohomology-generating' mechanism relies on the fact that the $s \ell(2)$-singular vectors are (upon dressing with ghosts appropriately) BRST-trivial, and therefore their BRST-primitives become cohomological states in the module where singular vectors vanish. In our case, combining these considerations with the existence of the spectral sequence, we will be able to give a more detailed structure of the cohomology. In the next section, we will consider how the cohomology of $s \ell(2)$ is generated from the state $|j\rangle_{\mathcal{A}}$ by the $N=4$ algebra operators. It looks plausible that the cohomology of $\mathcal{Q}^{(0)}=\oint \mathcal{C}_{0} \widehat{J}^{0}$ is generated precisely by the currents of the $N=4$ algebra.

\section{$3 \quad$ MFF vectors and cohomology}

In this section, we begin with the MFF singular states and then discuss how, upon an appropriate dressing with ghosts, they rewrite as $N=4$ singular states, and the further analysis can be carried out in terms of the $N=4$ algebra.

\subsection{MFF vectors and Verma modules at $k=-4$}

Consider singular states in the $s \ell(2)$ Verma module built on the highest-weight state $|j\rangle$. They are given by the MFF construction [5] and are labeled by two positive integers $r$ and $s$. For $j=j_{+}(r, s)($ see $(2.29))$ one has

$$
\begin{aligned}
|\operatorname{MFF}\{r, s\},-\rangle= & \left(J_{0}^{-}\right)^{r+(s-1)(k+2)}\left(J_{-1}^{+}\right)^{r+(s-2)(k+2)}\left(J_{0}^{-}\right)^{r+(s-3)(k+2)} \ldots \\
& \times\left(J_{-1}^{+}\right)^{r-(s-2)(k+2)}\left(J_{0}^{-}\right)^{r-(s-1)(k+2)}\left|j_{+}(r, s)\right\rangle
\end{aligned}
$$

The MFF states $|\mathrm{MFF}\{r, s\}\rangle$ are annihilated by the same set of annihilation operators as the highestweight $|j\rangle$ (see (2.11)) but have different spin and dimension (which for $k=-4$ are equal to $j-r$ and $-\frac{1}{2}(j-r)(j-r+1)$ respectively). 
Singular states determine the pattern of Verma module embeddings. In our case of $k=-4$, every Verma module contains only a finite number of submodules (corresponding to singular vectors) but can itself be embedded into an infinite number of other modules (cosingular vectors). Several lower- $j$ embeddings that correspond to singular vectors (3.1) are shown here:

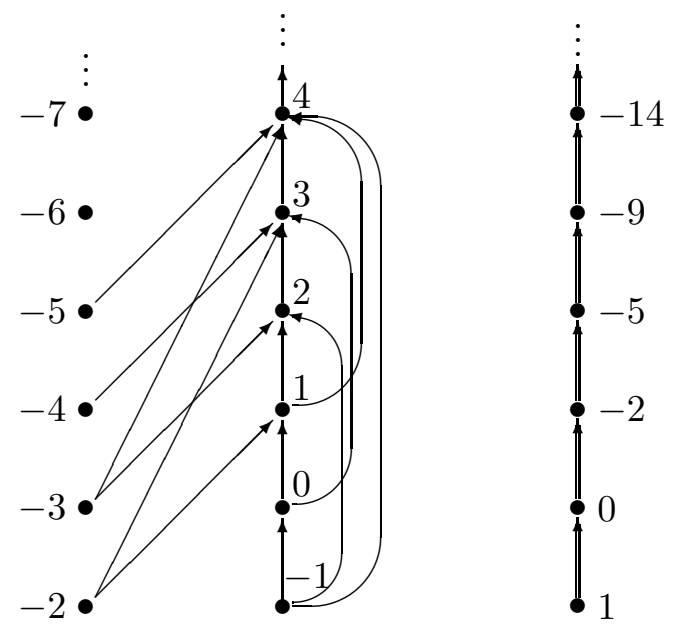

The numbers give the values of $j$; it should not be forgotten that there is an infinite number of arrows going out of any dot to the 'higher' ones. The right column with double arrows represents Virasoro Verma modules obtained via Hamiltonian reduction (the numbers give dimensions). The arrows are drawn in the direction of embeddings. There are precisely $j+1$ arrows entering a dot labelled by spin $j>0$. This pattern is determined by the fact that, for integral $j$ and negative integral $k$, there exist several ways to represent the spin $j$ as $j_{+}(r, s)$ with positive integral $r$ and $s$.

A 'dual' version of this embedding diagram exists for $j=j_{-}(r, s)$ (see (2.30)), based on the MFF vectors given by a construction similar to (3.1). The corresponding counterpart of (3.1) reads

$$
\begin{aligned}
|\operatorname{MFF}\{r, s\},+\rangle= & \left(J_{-1}^{+}\right)^{r+(s-1)(k+2)}\left(J_{0}^{-}\right)^{r+(s-2)(k+2)}\left(J_{-1}^{+}\right)^{r+(s-3)(k+2)} \ldots \\
& \times\left(J_{0}^{-}\right)^{r-(s-2)(k+2)}\left(J_{-1}^{+}\right)^{r-(s-1)(k+2)}\left|j_{-}(r, s)\right\rangle
\end{aligned}
$$

(with $k \rightarrow 4$ ). In what follows, we will mainly give explicit expressions for constructions related to the MFF vectors $|\operatorname{MFF}\{r, s\},-\rangle$, denoting them simply as $|\operatorname{MFF}\{r, s\}\rangle$.

\subsection{From $s \ell(2)_{-4}$ to $N=4$ singular vectors}

By dressing with ghosts, the $s \ell(2)$ singular vectors can be made into singular vectors in the $N=4$ representation considered above. Thus the BRST-primitives that represent the cohomology in the corresponding irreducible models, will be given by vectors in the $N=4$ representation; therefore the cohomology of $\mathcal{Q}_{\mathcal{A}}$ is concentrated in the $N=4$ term of the spectral sequence. Now that we have an (almost) explicit formula for the MFF singular vectors, it is interesting to see to what extent it can be carried over to the $N=4$ algebra that arises in the spectral sequence. Taking an MFF state and tensoring it with the ghost vacua from section 2 , as

$$
|\operatorname{MFF}\{r, s\}\rangle \otimes|j+1\rangle_{+} \otimes|0\rangle_{0} \otimes|1\rangle_{-}
$$


we observe that this can be dressed with ghost modes so as to produce a state with zero $\widehat{J}^{0}$-spin: for $j>0$ (with the formula (3.1) valid for $s \ell(2)$ singular vectors), the dressed states would read

$$
|\operatorname{MFF}\{r, s\}\rangle_{\mathcal{A}}= \begin{cases}|\mathrm{MFF}\{r, s\}\rangle \otimes \mathcal{B}_{j-r+1}^{+} \ldots \mathcal{B}_{j-1}^{+} \mathcal{B}_{j}^{+}|j+1\rangle_{+} \otimes|0\rangle_{0} \otimes|1\rangle_{-}, & r \leq j+1 \\ |\operatorname{MFF}\{r, s\}\rangle \otimes \mathcal{B}_{0}^{+} \ldots \mathcal{B}_{j-1}^{+} \mathcal{B}_{j}^{+}|j+1\rangle_{+} \otimes|0\rangle_{0} \otimes\left(\mathcal{C}_{-}\right)_{j-r+1} \ldots\left(\mathcal{C}_{-}\right)_{-1}|1\rangle_{-}, & r>j+1\end{cases}
$$

The states thus obtained turn out to be $\mathcal{Q}_{\mathcal{A}}$-closed and, moreover, $\mathcal{Q}_{\mathcal{A}}$-exact. Then, any state $|*\rangle$ such that

$$
|\operatorname{MFF}\{r, s\}\rangle_{\mathcal{A}}=\mathcal{Q}_{\mathcal{A}}|*\rangle
$$

would be a representative in the $\mathcal{Q}_{\mathcal{A}}$-cohomology of the irreducible module obtained by factorization of the Verma module over the null vector $|\operatorname{MFF}\{\mathrm{r}, \mathrm{s}\}\rangle$.

These cohomology elements occur in a particular term of the spectral sequence associated with (2.20), namely as states in the representation of the $N=4$ algebra from the previous section. They can indeed be constructed by acting with modes of the $N=4$ generators (2.22) on the vacuum $|\mathrm{j},+\rangle_{N=4}$ (similarly, for $j<0$, the corresponding MFF vectors (3.3) can be dressed with ghosts in such a way that would allow rewriting them as $N=4$ singular vectors built on the vacuum $|\mathrm{j},-\rangle)$. For definiteness, we will consider explicitly the $N=4$ singular vectors built on the $|\mathrm{j},+\rangle$ vacua.

Consider first the $r 1 \mathrm{MFF}$ states. They are built on the $s \ell(2)$ highest-weight state of spin $j=\frac{1}{2}(r-1)$ and can thus be written as $|\operatorname{MFF}\{2 j+1,1\}\rangle$. When dressed with the ghosts, the states $|\operatorname{MFF}\{2 j+1,1\}\rangle_{\mathcal{A}}$ are identically rewritten as elements of the $(N=4)_{1}$ representation, i.e. generated from the vacuum by the action of the $N=4$ generators:

$$
|\operatorname{MFF}\{2 \mathrm{j}-1,1\}\rangle_{\mathcal{A}}=\left(\overline{\mathcal{G}}_{2}\right)_{-\mathrm{j}+2}\left(\overline{\mathcal{G}}_{2}\right)_{-\mathrm{j}+3} \ldots\left(\overline{\mathcal{G}}_{2}\right)_{-1}\left(\overline{\mathcal{G}}_{1}\right)_{0} \ldots\left(\overline{\mathcal{G}}_{1}\right)_{\mathrm{j}-2}|\mathrm{j},+\rangle_{N=4}
$$

Moreover, these states being $\left(\overline{\mathcal{G}}_{2}\right)_{0}$-exact, the corresponding BRST-primitive state $|*\rangle$ in (3.6) can be obtained by pulling out the BRST operator $\mathcal{Q}^{(1)}=\left(\overline{\mathcal{G}}_{2}\right)_{0}$. We then arrive at the following representation for the primitives in terms of $N=4$ algebra generators acting on the vacuum:

$$
|*\rangle=\left(J_{N=4}^{0}\right)_{-\mathbf{j}+2}\left(\overline{\mathcal{G}}_{2}\right)_{-\mathrm{j}+3} \ldots\left(\overline{\mathcal{G}}_{2}\right)_{-1}\left(\overline{\mathcal{G}}_{1}\right)_{0} \ldots\left(\overline{\mathcal{G}}_{1}\right)_{\mathrm{j}-2}|\mathrm{j},+\rangle_{N=4}
$$

(as compared with (3.7), the leftmost mode $\left(\overline{\mathcal{G}}_{2}\right)_{m}$ gets replaced by the same mode of $\left.J_{N=4}^{0}\right)$. This expression thus gives an element in cohomology upon factorization over the submodule generated by the MFF vector.

More generally, consider an arbitrary MFF vector for $k=-4$ with the only condition that all the powers in the MFF formula (3.1) be non-negative. Among the $|\mathrm{MFF},-\rangle$-vectors these are $|\mathrm{MFF}\{j+l, s\}\rangle$ for $1 \leq l \leq j+1$. To elucidate their construction in terms of the $N=4$ algebra

\footnotetext{
${ }^{4}$ more precisely, $|\operatorname{MFF}\{2 j+1,1\},-\rangle$; the ' + '-counterpart reads $|\operatorname{MFF}\{k+1-2 j, 1\},+\rangle$.
} 
generators, we write them down together with the corresponding MFF formula:

$$
\begin{array}{cc}
|\mathrm{MFF}\{2 \mathrm{j}-s-1, s\},-\rangle_{\mathcal{A}}= & |\mathrm{MFF}\{2 \mathrm{j}-s-1, s\}\rangle= \\
\left(\overline{\mathcal{G}}_{2}\right)_{-\mathrm{j}+s} \ldots\left(\overline{\mathcal{G}}_{2}\right)_{-1}\left(\overline{\mathcal{G}}_{1}\right)_{0} \ldots\left(\overline{\mathcal{G}}_{1}\right)_{\mathbf{j}-s} & \left(J_{0}^{-}\right)^{2 \mathrm{j}-2 s+1} \\
\left(\mathcal{G}^{1}\right)_{-\mathrm{j}+s-1} \ldots\left(\mathcal{G}^{1}\right)_{-1}\left(\mathcal{G}^{2}\right)_{0} \ldots\left(\mathcal{G}^{2}\right)_{-\mathrm{j}-s+1} & \left(J_{-1}^{+}\right)^{2 \mathrm{j}-2 s+3} \\
\vdots & \vdots \\
\left(\overline{\mathcal{G}}_{2}\right)_{-\mathrm{j}+4} \ldots\left(\overline{\mathcal{G}}_{2}\right)_{-1}\left(\overline{\mathcal{G}}_{1}\right)_{0} \ldots\left(\overline{\mathcal{G}}_{1}\right)_{\mathbf{j}-4} & \left(J_{0}^{-}\right)^{2 \mathrm{j}-7} \\
\left(\mathcal{G}^{1}\right)_{-\mathrm{j}+3} \ldots\left(\mathcal{G}^{1}\right)_{-1}\left(\mathcal{G}^{2}\right)_{0} \ldots\left(\mathcal{G}^{2}\right)_{\mathbf{j}-3} & \left(J_{-1}^{+}\right)^{2 \mathrm{j}-5} \\
\left(\overline{\mathcal{G}}_{2}\right)_{-\mathbf{j}+2} \ldots\left(\overline{\mathcal{G}}_{2}\right)_{-1}\left(\overline{\mathcal{G}}_{1}\right)_{0} \ldots\left(\overline{\mathcal{G}}_{1}\right)_{\mathbf{j}-2}|\mathbf{j},+\rangle_{N=4} & \left(J_{0}^{-}\right)^{2 \mathbf{j}-3}|\mathbf{j}-2\rangle
\end{array}
$$

The $N=4$ spin (the eigenvalue of $2\left(J_{N=4}^{0}\right)_{0}$ ) of this state is equal to $l=\mathrm{j}-2 s+1$. Every MFF factor $\left(J_{-1,0}^{ \pm}\right)^{m}$ corresponds to a group of $m$ factors given by modes of the $N=4$ generators. The groups corresponding to $\left(J_{0}^{-}\right)^{m}$ consist of $\frac{1}{2}(m+1)$ generators $\overline{\mathcal{G}}_{1}$, their modes ranging from $\frac{1}{2}(m-1)$ to 0 (recall that $m$ is always odd). In addition, the same group contains a product of modes of $\overline{\mathcal{G}}_{2}$, from $\left(\overline{\mathcal{G}}_{2}\right)_{-1}$ to $\left(\overline{\mathcal{G}}_{2}\right)_{\frac{-m+1}{2}}$. Thus, when passing the zero mode, the $N=4$ generators inside one group get replaced according to the action of the automorphism (2.27) (however the left subgroup is one element shorter). To obtain the structure of the groups of $N=4$ factors corresponding to $\left(J_{-1}^{+}\right)^{m}$, one should drop the leftmost and the rightmost modes in the right neighbouring group (the one corresponding to $\left(J_{0}^{-}\right)^{m+2}$ ) and then act on the remaining modes with the Weyl reflection (2.26).

To check that the states constructed are singular vectors in the $N=4$ module, consider the state obtained by acting on $|\mathrm{j},+\rangle_{N=4}$ with only the first (counting from the right) group in (3.9) (the case $s=1$ ),

$$
\left(\overline{\mathcal{G}}_{2}\right)_{-\mathrm{j}+2} \ldots\left(\overline{\mathcal{G}}_{2}\right)_{-1}\left(\overline{\mathcal{G}}_{1}\right)_{0} \ldots\left(\overline{\mathcal{G}}_{1}\right)_{\mathrm{j}-2}|\mathrm{j},+\rangle_{N=4} .
$$

The eigenvalue of $2\left(J_{N=4}^{0}\right)_{0}$ on $(3.10)$ is given by $\mathrm{j}-\left(\#\right.$ of $\left.\overline{\mathcal{G}}_{1}\right)+\left(\#\right.$ of $\left.\overline{\mathcal{G}}_{2}\right)=\mathrm{j}-1$. The state (3.10) is in fact a $|\mathrm{j}-1,-\rangle_{N=4}$. To see this, note first of all that it is annihilated by $\left(\overline{\mathcal{G}}_{1}\right)_{\geq 0}$, since the modes $\left(\overline{\mathcal{G}}_{1}\right)_{\geq \mathrm{j}-1}$ used to annihilate $|\mathrm{j},+\rangle_{N=4}$ while the remaining modes $\mathcal{G}_{0 \leq n \leq \mathrm{j}-2}$ will square to zero. Similarly, 3.10) is annihilated by $\left(\overline{\mathcal{G}}_{2}\right)_{\geq-j+2}$. This gives a half of the highest-weight conditions (2.34) for spin $\mathrm{j}-1$. The other half can be deduced as follows.

Let us evaluate the action of $\mathcal{G}_{n}^{1}$ on (3.10) for $n \geq 0$. When being plugged to the right, $\mathcal{G}_{n}^{1}$ can hit one of the $\overline{\mathcal{G}}_{2}$ or one of the $\overline{\mathcal{G}}_{1}$ modes. Consider first

$$
\left[\mathcal{G}_{n}^{1},\left(\overline{\mathcal{G}}_{2}\right)_{-\mathrm{j}+2} \ldots\left(\overline{\mathcal{G}}_{2}\right)_{-1}\right]\left(\overline{\mathcal{G}}_{1}\right)_{0} \ldots\left(\overline{\mathcal{G}}_{1}\right)_{\mathrm{j}-2}|\mathrm{j},+\rangle_{N=4}
$$

Using the $N=4$ commutation relations that follow from (2.23) (the brackets [, ] always mean the supercommutator)

$$
\begin{aligned}
{\left[\mathcal{G}_{n}^{a},\left(\overline{\mathcal{G}}_{b}\right)_{m}\right]=} & \delta_{b}^{a}(n+a-1)(n+a-2) \delta_{m+n, 0}+(m-n+3-a-b)\left(\sigma^{\alpha}\right)_{b}^{a} \eta_{\alpha \beta}\left(J_{N=4}^{\beta}\right)_{m+n} \\
& +\delta_{b}^{a} \mathcal{L}_{m+n}+(m+n+1) \delta_{b}^{a}\left(J_{N=4}^{0}\right)_{m+n},
\end{aligned}
$$

we see that $\left[\mathcal{G}_{n}^{1},\left(\overline{\mathcal{G}}_{2}\right)_{m}\right]=(m-n)\left(J_{N=4}^{+}\right)_{m+n}$ and the resulting $\left(J_{N=4}^{+}\right)_{m+n}$ can be moved to the right until it meets $\left(\overline{\mathcal{G}}_{1}\right)_{0}$. Commuting $\left(J_{N=4}^{+}\right)_{m+n}$ with the modes $\left(\overline{\mathcal{G}}_{1}\right)_{r}$ will produce $\left(\overline{\mathcal{G}}_{2}\right)_{n+m+r}$. Of these, $\left(\overline{\mathcal{G}}_{2}\right)_{p}$ with $p \geq 0$ will annihilate the state $|\mathbf{j},+\rangle_{N=4}$, while those with $-\mathbf{j}+2 \leq p \leq-1$ will square to zero due to the presence of the same mode among the $\left(\overline{\mathcal{G}}_{2}\right)_{-\mathbf{j}+2} \ldots\left(\overline{\mathcal{G}}_{2}\right)_{-1}$ unless 
this mode has been 'spent' in the commutator $\left[\mathcal{G}_{n}^{1},\left(\overline{\mathcal{G}}_{2}\right)_{m}\right]$; however, that would never happen for $n>0$, and thus the result of commuting $\mathcal{G}_{n}^{1}$ through the $\left(\overline{\mathcal{G}}_{2}\right)_{-\mathbf{j}+2} \ldots\left(\overline{\mathcal{G}}_{2}\right)_{-1}$ is effectively zero for $n>0$. When $n=0$, however, the mode $\left(\overline{\mathcal{G}}_{2}\right)_{m}$ will be restored when commuting $\left(J_{N=4}^{+}\right)_{m}$ with $\left(\overline{\mathcal{G}}_{1}\right)_{0}$, which gives

$$
\begin{aligned}
& {\left[\mathcal{G}_{0}^{1},\left(\overline{\mathcal{G}}_{2}\right)_{-\mathrm{j}+2} \ldots\left(\overline{\mathcal{G}}_{2}\right)_{-1}\right]\left(\overline{\mathcal{G}}_{1}\right)_{0} \ldots\left(\overline{\mathcal{G}}_{1}\right)_{\mathbf{j}-2}|\mathbf{j},+\rangle_{N=4}} \\
& \quad=\sum_{m=-\mathrm{j}+2}^{-1} m(-1)^{\mathrm{j}+m}\left(\overline{\mathcal{G}}_{2}\right)_{-\mathbf{j}+2} \ldots\left(\overline{\mathcal{G}}_{2}\right)_{m} \ldots\left(\overline{\mathcal{G}}_{2}\right)_{-1} \cdot\left(\overline{\mathcal{G}}_{2}\right)_{m}\left(\overline{\mathcal{G}}_{1}\right)_{1} \ldots\left(\overline{\mathcal{G}}_{1}\right)_{\mathbf{j}-2}|\mathbf{j},+\rangle_{N=4} \\
& \quad=\frac{1}{2}(-1)^{\mathrm{j}}(\mathrm{j}-1)(\mathrm{j}-2)\left(\overline{\mathcal{G}}_{2}\right)_{-\mathbf{j}+2} \ldots\left(\overline{\mathcal{G}}_{2}\right)_{-1} \cdot\left(\overline{\mathcal{G}}_{1}\right)_{1} \ldots\left(\overline{\mathcal{G}}_{1}\right)_{\mathrm{j}-2}|\mathrm{j},+\rangle_{N=4}
\end{aligned}
$$

It remains to see how $\mathcal{G}_{n}^{1}$ commutes with $\left(\overline{\mathcal{G}}_{1}\right)_{0} \ldots\left(\overline{\mathcal{G}}_{1}\right)_{\mathrm{j}-2}$, namely to evaluate

$$
(-1)^{\mathrm{j}}\left(\overline{\mathcal{G}}_{2}\right)_{-\mathrm{j}+2} \ldots\left(\overline{\mathcal{G}}_{2}\right)_{-1}\left[\mathcal{G}_{n}^{1},\left(\overline{\mathcal{G}}_{1}\right)_{0} \ldots\left(\overline{\mathcal{G}}_{1}\right)_{\mathrm{j}-2}\right]|\mathrm{j},+\rangle_{N=4}
$$

Here, in accordance with $(2.23)$

$$
\left[\mathcal{G}_{n}^{1},\left(\overline{\mathcal{G}}_{1}\right)_{m}\right]=n(n-1) \delta_{m+n, 0}+2 n\left(J_{N=4}^{0}\right)_{m+n}+\mathcal{L}_{m+n}
$$

and for $n=0$ we find $\left[\mathcal{G}_{0}^{1},\left(\overline{\mathcal{G}}_{1}\right)_{0}\right]=\mathcal{L}_{0}$; plugging $\mathcal{L}_{0}$ to the right amounts to adding up the mode numbers of $\left(\overline{\mathcal{G}}_{1}\right)_{1} \ldots\left(\overline{\mathcal{G}}_{1}\right)_{\mathrm{j}-2}$ as

$$
\sum_{r=1}^{\mathrm{j}-2}(-r)=-\frac{1}{2}(\mathrm{j}-1)(\mathrm{j}-2),
$$

and the resulting term will precisely cancel (3.13). All other commutators in (3.14) for $n=0$ give a vanishing contribution. For $n \geq 1$, (3.14) vanishes altogether.

Analyzing similarly the action of the modes $\left(J_{N=4}^{+}\right)_{n \geq-\mathrm{j}+2},\left(\mathcal{G}^{2}\right)_{n \geq \mathrm{j}}$, and $\left(J_{N=4}^{-}\right)_{n \geq \mathrm{j}}$ on (3.10), we also find that these are annihilators. The necessary highest-weight relations (see (2.34)) require in addition two more vanishing conditions, namely those for $\left(J_{N=4}^{-}\right)_{\mathrm{j}-1}$ and $\left(\mathcal{G}^{2}\right)_{\mathrm{j}-1}$. These are satisfied by virtue of the relations (2.38).

We thus conclude that the state $\left(\overline{\mathcal{G}}_{2}\right)_{-\mathrm{j}+2} \ldots\left(\overline{\mathcal{G}}_{2}\right)_{-1}\left(\overline{\mathcal{G}}_{1}\right)_{0} \ldots\left(\overline{\mathcal{G}}_{1}\right)_{\mathrm{j}-2}|\mathrm{j},+\rangle_{N=4}$ is indeed proportional to $|\mathrm{j}-1,-\rangle_{N=4}$. Then the second from the right group in (3.9) maps the resulting vector into $|\mathrm{j}-2,+\rangle_{N=4}$ etc., which can be shown either directly or simply by noticing that the $N=4$ generators in the adjacent groups are related by the Weyl reflection (2.26) (and the groups become shorter as moving from right to left, according to how the $J_{N=4}^{0}$-spin decreases). The vectors (3.9) are therefore singular in our $(N=4)_{1}$ module.

To compare with the formulation of ref. [23], we notice that a singular vector in the module built over the 'proper' highest weight (2.35) can be constructed by means of the following procedure. One dresses the state $\left|\frac{1}{2}, \Delta\right\rangle_{N=4}(2.35)$ with $\Delta=-\frac{1}{2} \mathrm{j}(\mathrm{j}-1)$ using the formula 2.36) and obtains a $|\mathrm{j},+\rangle$; then one builds over $|\mathrm{j},+\rangle$ the singular vector (3.9) and obtains $|\operatorname{MFF}\{2 \mathrm{j}-s-1, s\},-\rangle_{\mathcal{A}}$. And finally dressing it as in (2.37) one obtains a new highest weight obeying (2.35) with a new dimension $\Delta=-\frac{1}{2}(\mathrm{j}-2 s)(\mathrm{j}-2 s-1)$. Therefore, non-unitary $(N=4)_{1}$ modules are embedded into one another according to the pattern described above, with the massless representation $(\Delta=0)$ being embedded into all the other.

\footnotetext{
${ }^{5}$ and the relations (2.38) are Weyl-symmetric as well.
} 
The $\mathcal{Q}_{\mathcal{A}}$-primitives of the singular states thus constructed are given in the same way as in (3.8), hence follow representatives in the cohomology of the irreducible modules.

Note that once the Weyl group action on the $N=4$ generators has been identified, the mechanism behind the above derivation is very similar to the one underlying the MFF construction. The analogies with the $s \ell(2) \mathrm{MFF}$ formula are rather straightforward, however we have not carried out an 'analytic' continuation of the construction (3.9) off the positive integer points. Recall that the power of the MFF construction is that it can be given meaning for all values of the parameters, when the exponents in (3.1) are complex numbers. The $N=4$ analogue of the 'continued' formula would require introducing operators with non-integer modding (which is the easy part of the construction) and replacing the products of modes with intertwiners (which is somewhat more involved). We actually expect a close analogy with the case of (twisted) $N=2$ algebra, for which the 'analytically' (in fact, algebraically) continued construction can indeed be built [30]. Yet we have not tried to extend, in $N=4$ terms, the formula (3.9) to the case of $r$ and $s$ being arbitrary positive integers. We have only checked in a number of lower-level cases that the MFF singular vectors taken in the polynomial form do rewrite, upon dressing with ghosts, as $N=4$ descendants. That they satisfy $N=4$ highest-weight conditions can be proven in general, and therefore we conjecture a 1:1 correspondence between $s \ell(2)_{k=-4}$ singular vectors and those in the $(N=4)_{k=1}$ module. As explained above, they give rise to cohomology in factor-modules.

\section{$4 \mathcal{Q}^{(0)}$ cohomology by bosonization}

We have observed the $N=4$ algebra in the cohomology of $\mathcal{Q}^{(0)}$. It is very useful to 'straighten' the construction by explicitly projecting onto the cohomology of $\mathcal{Q}^{(0)}$. This amounts to projecting out (normal-ordered) operator monomials that contain $\mathcal{B}^{0}$ and $\widehat{J}^{0}$ (and their derivatives), since $\mathcal{B}^{0}$ is not $\mathcal{Q}^{(0)}$-closed while $\widehat{J}^{0}$ is $\mathcal{Q}^{(0)}$-exact (see $(2.7),(2.8)$ ). Effectively solving the constraint $\widehat{J}^{0} \sim 0$ will now be achieved by using a particular representation of the $s \ell(2)$ currents and will result in a 'strong' $N=4$ algebra (rather than the one modulo $\mathcal{Q}^{(0)}$-exact terms).

\subsection{Bosonizing $s \ell(2)$ currents and ghosts}

In this subsection, we will introduce a representation of the $s \ell(2)$ currents and the associated ghosts that would allow us to extract the $N=4$ algebra at the level of operator products, not only in cohomology.

We start with the representation from ref. [7] which is 'induced' from a Verma module of a minimal conformal theory. Generally, this representation can be considered as an embedding $s \ell(2) \hookrightarrow \mathcal{M}_{d} \oplus \mathcal{L} \oplus[B, C] \oplus U(1)_{v}$ where $\mathcal{M}_{d}$ denotes the minimal model, $\mathcal{L}$ is the theory of a free scalar $\varphi,[B, C]$ is a ghost system, and $U(1)_{v}$ is an additional scalar theory of a field $v$ whose signature is opposite to that of $\varphi$. The minimal-model central charge $d$ is taken to satisfy the hamiltonian reduction formula 25

$$
d=13-6(k+2)-\frac{6}{k+2}
$$

where $k$ is the level of the $s \ell(2)$ algebra. Setting, in our case, $k=-4$, leads to $d=28$. Such a $d=28$-model is naturally viewed as a 'Liouville' theory; similarly, the scalar $\varphi$ that used to be a 'Liouville' in ref. [7] is redefined by absorbing the imaginary unit into it, which gives $\varphi$ a matter-like signature (and restores, in our conventions, real background charge). The signature of the additional 
scalar $\partial v$ changes similarly from matter-like in the formulae of [7] to Liouville-like. After all these redefinitions, the formulae of ref. [0] for $s \ell(2)_{-4}$ currents take the form

$$
\begin{aligned}
& J^{+}=e^{\varphi-v}, \quad J^{0}=B C-\partial \varphi+2 \partial v, \\
& J^{-}=\left(T_{m}+\frac{1}{2} \partial \varphi \partial \varphi-\frac{3}{2} \partial^{2} \varphi-\partial B C-2 B \partial C+B C \partial \varphi\right) e^{-(\varphi-v)}
\end{aligned}
$$

where

$$
v(z) v(w)=-\log (z-w), \quad \varphi(z) \varphi(w)=\log (z-w) .
$$

The ghost, $\varphi$ - and $v$ - energy-momentum tensors read

$$
T_{B C}=-B \partial C, \quad T_{\varphi}=\frac{1}{2}(\partial \varphi)^{2}-\frac{3}{2} \partial^{2} \varphi, \quad T_{v}=-\frac{1}{2}(\partial v)^{2}+\frac{3}{2} \partial^{2} v
$$

Then the currents $J^{+}, J^{0}$, and $J^{-}$are given dimensions 0,1 , and 2 respectively with respect to the energy-momentum tensor $T_{B C}+T_{\varphi}+T_{v}+T_{\mathrm{m}}$.

In addition to the $s \ell(2)$ currents, the system of $T_{\mathrm{m}}, \varphi, v$ and $B C$ ghosts allows one to represent an independent fermionic ghost system, which we are going to identify with $\mathcal{B}^{-} \mathcal{C}_{-}$:

$$
\mathcal{B}^{-}=B e^{-(\varphi-v)}, \quad \mathcal{C}_{-}=C e^{\varphi-v}
$$

Evaluating the twisted Sugawara and $\mathcal{B}^{-} \mathcal{C}_{-}$ghosts' energy-momentum tensors in terms of the 'elementary' fields $T_{\mathrm{m}}, \varphi, v$ and $B C$, we arrive at the relation

$$
\widetilde{T^{\mathrm{S}}}-\mathcal{B}^{-} \partial \mathcal{C}_{-}=T_{\mathrm{m}}+\frac{1}{2}(\partial \varphi)^{2}-\frac{3}{2} \partial^{2} \varphi-B \partial C-\frac{1}{2}(\partial v)^{2}+\frac{3}{2} \partial^{2} v
$$

which shows that we have an equal number of degrees of freedom in the $\mathcal{M}_{d} \oplus \mathcal{L} \oplus[B, C] \oplus U(1)_{v}$ system and in the $s \ell(2)_{-4}$ currents with one ghost pair $\left(\mathcal{B}^{-} \mathcal{C}_{-}\right)$.

In section 2, however, we had more ghost pairs. Our next objective is to extend the representation (4.2), (4.5) so as to incorporate the other ghosts and then derive a realization for the $N=4$ algebra. This would require changing coordinates on the field space so as to effectively solve the constraint $\widehat{J}^{0} \sim 0$. To this end, we first notice that since $\widehat{J}^{0}$ is OPE-isotropic, $\widehat{J}^{0}(z) \widehat{J}^{0}(w)=0$, it can be represented by a complex scalar $\partial \phi$, with

$$
\phi(z) \bar{\phi}(w)=\log (z-w), \quad \widehat{J}^{0}=\partial \phi .
$$

In the formulae (4.2) and (4.5), we did have an isotropic combination $\varphi-v$, and from the $s \ell(2)$ algebra we see that this must be conjugate to $\partial \phi=\widehat{J}^{0}$. We thus take $\bar{\phi}$ to be equal to $\varphi-v$, which allows us to have $J^{+}=\exp \bar{\phi}$. Next we extend the field space by an independent ghost system denoted by $b c$, and express $\left(\partial v, \partial \varphi, \mathcal{B}^{+}, \mathcal{C}_{+}\right)$through $(\partial \phi, \partial \bar{\phi}, b, c)$ via

$$
\begin{aligned}
\partial v & =b c+\partial \phi-\partial \bar{\phi} \\
\partial \varphi & =b c+\partial \phi, \\
\mathcal{B}^{+} & =c e^{\bar{\phi}}, \quad \mathcal{C}_{+}=b e^{-\bar{\phi}}
\end{aligned}
$$

after which the $s \ell(2)_{-4}$ currents take the form:

$$
\begin{aligned}
J^{+} & =e^{\bar{\phi}} \\
J^{0} & =b c+B C+\partial \phi-2 \partial \bar{\phi} \\
J^{-} & =\left(T_{\mathrm{m}}+\frac{1}{2} \partial \phi \partial \phi-\frac{3}{2} \partial^{2} \phi+b c \partial \phi+B C \partial \phi-2 b \partial c-\partial b c-2 B \partial C-\partial B C+B C b c\right) e^{-\bar{\phi}}
\end{aligned}
$$


while the $\mathcal{B}^{+} \mathcal{C}_{+}$and $\mathcal{B}^{-} \mathcal{C}_{-}$ghosts are now given by:

$$
\begin{array}{ll}
\mathcal{B}^{-}=B e^{-\bar{\phi}}, & \mathcal{C}_{-}=C e^{\bar{\phi}} \\
\mathcal{B}^{+}=c e^{\bar{\phi}}, & \mathcal{C}_{+}=b e^{-\bar{\phi}} .
\end{array}
$$

Note that the $\mathcal{B}^{0} \mathcal{C}_{0}$ ghosts 'decouple' - they do not participate in field redefinitions.

Using formulae (4.11), (4.12), we can now evaluate the energy-momentum tensor (2.3) in terms of the new fields $\phi, \bar{\phi}, b c, B C, T_{\mathrm{m}}$ :

$$
\mathcal{T}_{\mathcal{A}}=\underbrace{T_{\mathrm{m}}-b \partial c-2 B \partial C-\partial B C}+\partial \phi \partial \bar{\phi}-\partial^{2} \phi-\mathcal{B}^{0} \partial \mathcal{C}_{0}
$$

It follows that $\operatorname{dim} B=2$ while $\operatorname{dim} b=1 ; b c$ thus represent a $c=-2$ matter and together with $T_{\mathrm{m}}$ (which plays the rôle of a Liouville) and the $B C$ ghosts make up a realization of the bosonic string.

\subsection{Constructing the states}

Let us see now how the representation space can be constructed for the realization (4.11), 4.12). The vacuum is found by translating the vacuum determined by (2.11), 2.16) to the present picture. This gives for (2.12) (assuming $j>0$ for definiteness)

$$
|j\rangle \otimes \underbrace{|j+1\rangle_{+} \otimes|0\rangle_{0} \otimes|1\rangle_{-}}_{\mathcal{B C} \text { ghosts }}=\underbrace{|\Delta(r, s)\rangle_{\mathrm{m}} \otimes|-j-1\rangle_{b c} \otimes|1\rangle_{B C}}_{c=-2 \text { bosonic string }} \otimes|0\rangle_{0} \otimes|\{0,0\}\rangle_{\phi \bar{\phi}}
$$

where $|\{0,0\}\rangle_{\phi \bar{\phi}}$ is the trivial vacuum in the complex scalar theory. The $b c$ and $B C$ ghost vacua from the RHS of (4.14) are characterized by creation/annihilation conditions depending (for $b c$ ) on the $s \ell(2)$ spin $j$.

$$
\begin{aligned}
& c_{n}|-j-1\rangle_{b, c}=0 \quad n \geq j+2, \quad b_{n}|-j-1\rangle_{b, c}=0 \quad n \geq-j-1, \\
& C_{n}|1\rangle_{B, C} \quad=0 \quad n \geq 1, \quad B_{n}|1\rangle_{B, C} \quad=0 \quad n \geq 0 \text {. }
\end{aligned}
$$

while the conformal dimension of the primary matter state $|\Delta(r, s)\rangle_{\mathrm{m}}$ is taken from the Kač table for $d=28$ :

$$
\Delta(r, s)=\frac{1}{4}\left(-\frac{1}{2}\left(r^{2}-1\right)-2\left(s^{2}-1\right)-2 r s+2\right)=-\frac{1}{8}\left((r+2 s)^{2}-9\right)
$$

which rewrites as

$$
\Delta(r, s)=-\frac{1}{2} j(r, s)(j(r, s)+3)
$$

where $j(r, s)=j_{+}(r, s)$, i.e.

$$
j(r, s)=\frac{r-1}{2}+2 \frac{s-1}{2} \quad r \text { and } s \text { are integer }
$$

For $j=j_{-}(r, s)$, we can do by analogy with the previous case, for example the formula (4.14) rewrites as

$$
|j\rangle \otimes \underbrace{|0\rangle_{+} \otimes|0\rangle_{0} \otimes|-j\rangle_{-}}_{\mathcal{B C} \text { ghosts }}=\underbrace{|\Delta(r, s)\rangle_{\mathrm{m}} \otimes|0\rangle_{b c} \otimes|-j\rangle_{B C}}_{c=-2 \text { bosonic string }} \otimes|0\rangle_{0} \otimes|\{0,0\}\rangle_{\phi \bar{\phi}}
$$


The formula (4.17) for the dimension of the matter state $|\Delta(r, s)\rangle_{\mathrm{m}}$ remains the same.

As mentioned after the formula (2.37), it is possible to build the $N=4$ states $\left|\frac{1}{2}, \Delta\right\rangle_{N=4}$ in terms of matter $|\Delta(r, s)\rangle_{\mathrm{m}}$ and ghosts $B C$ and $b c$; the explicit formula reads

$$
\left|\frac{1}{2}, \Delta\right\rangle_{N=4}=|\Delta(r, s)\rangle_{\mathrm{m}} \otimes|0\rangle_{b c} \otimes|1\rangle_{B C}
$$

where $\Delta=\Delta(r, s)-1$, with -1 being accounted for by a $c$-ghost contribution.

The subspace built on $|\Delta(r, s)\rangle_{\mathrm{m}} \otimes|-j-1\rangle_{b c} \otimes|1\rangle_{B C}$ can be thought of as the space of states of a non-critical bosonic string with $c=-2$ matter. We are going to consider it in more detail.

\subsection{Bosonization of spectral sequence and the LZ states}

Now we are going to translate the spectral sequence associated with the decomposition (2.19) to the representation in terms of the 'elementary' fields $\phi, \bar{\phi}, b c, B C, T_{\mathrm{m}}$. These latter have then to be assigned the following degrees (cf. (2.18)):

$$
\begin{aligned}
& \operatorname{deg} T_{\mathrm{m}}=\operatorname{deg} \mathcal{C}_{0}=\operatorname{deg} \mathcal{B}^{0}=\operatorname{deg} \partial \phi=\operatorname{deg} \partial \bar{\phi}=0, \\
& \operatorname{deg} C=-\operatorname{deg} B=\operatorname{deg} e^{-\bar{\phi}}=-\operatorname{deg} e^{\bar{\phi}}=1, \\
& \operatorname{deg} b=-\operatorname{deg} c=2 .
\end{aligned}
$$

The different parts (2.20) of the BRST current now take the form

$$
\begin{aligned}
\mathcal{J}^{(0)} & =\mathcal{C}_{0} \partial \phi, \\
\mathcal{J}^{(1)} & =C\left(T_{\mathrm{m}}-b \partial c\right)-C B \partial C+\partial^{2} C-\partial(C b c)+C\left(\frac{1}{2} \partial \phi \partial \phi-\frac{1}{2} \partial^{2} \phi+\partial \phi b c\right)-\partial(\partial \phi C), \\
\mathcal{J}^{(2)} & =b, \\
\mathcal{J}^{(3)} & =b C \mathcal{B}^{0} .
\end{aligned}
$$

We can thus project onto the cohomology of $\mathcal{Q}^{(0)}$. Observe that the BRST current $\mathcal{J}^{(1)}$ and the energy-momentum tensor (4.13) can be rewritten as

$$
\begin{aligned}
& \mathcal{T}_{\mathcal{A}}=\mathcal{T}_{\text {str }}+\left[\mathcal{Q}^{(0)}, \mathcal{B}^{0} \partial \bar{\phi}-\partial^{2} \mathcal{B}^{0}\right] \\
& \mathcal{J}^{(1)}=\mathcal{J}_{\text {str }}+\left[\mathcal{Q}^{(0)}, \frac{1}{2} \mathcal{B}^{0}(C \partial \phi+C b c-2 \partial C)-\frac{3}{2} \partial \mathcal{B}^{0}\right]
\end{aligned}
$$

where fields in the cohomology can be taken as

$$
\begin{aligned}
& \mathcal{T}_{\text {str }}=T_{\mathrm{m}}-b \partial c-2 B \partial C-\partial B C, \\
& \mathcal{J}_{\text {str }}=C\left(T_{\mathrm{m}}-b \partial c\right)-C B \partial C+\partial^{2} C-\partial(C b c) .
\end{aligned}
$$

These are identified with the energy-momentum tensor and the BRST current of a bosonic string with a $c=-2$ matter represented by the $b c$ system.

Further, along with $\mathcal{T}_{\mathcal{A}}$ and $\mathcal{J}_{\mathcal{A}}$, the $N=4$ generators $(2.22)$ can be projected onto the $\mathcal{Q}^{(0)}$ cohomology. This will produce a 'strong' $N=4$ algebra, i.e. relations (2.23) will be satisfied exactly 
rather than modulo $\mathcal{Q}^{(0)}$-exact terms as was the case with the generators (2.22). Namely, the $N=4$ generators now take the form

$$
\begin{aligned}
\mathcal{T} & =\mathcal{T}_{\text {str }}, & \mathcal{G}^{1} & =b, \\
J_{N=4}^{+} & =C b, & \mathcal{G}^{2} & =B, \\
J_{N=4}^{0} & =\frac{1}{2}(b c-B C), & \overline{\mathcal{G}}_{1} & =c\left(T_{\mathrm{m}}-B \partial C\right)+b c \partial c-\partial(c B C)+\partial^{2} c, \\
J_{N=4}^{-} & =B c, & \overline{\mathcal{G}}_{2} & =\mathcal{J}_{\text {str }}
\end{aligned}
$$

Here, $\mathcal{T}=\mathcal{T}_{\text {str }}$ and $\overline{\mathcal{G}}_{2}=\mathcal{J}_{\text {str }}$ are given by eqs. (4.24), while $\mathcal{G}^{2}$ plays the rôle of a superpartner to $\mathcal{T}$. The system described by the energy-momentum tensor $\mathcal{T}_{\text {str }}$ represents a $c=-2$ matter coupled to gravity, with $T_{\mathrm{m}}$ playing (in accordance with the value of its central charge) the rôle of a Liouville.

Cohomology of the bosonized $\mathcal{Q}^{(0)}$ operator coincides with the $N=4$ algebra representation built on the $(N=4)$ highest-weight state obtained by dressing the string vacuum $|\Delta(r, s)\rangle_{\mathrm{m}} \otimes|0\rangle_{b c} \otimes|0\rangle_{B C}$ with the appropriate number of ghosts, eq. (4.20).

The BRST-primitives of the states (3.9) can now be written as BRST-primitives w.r.t. the BRST operator $\mathcal{Q}_{\text {str }}=\oint \mathcal{J}_{\text {str }}$. An important fact is that the MFF singular vectors (3.9) take in our representation the form (see [7] where this claim was based on using the (general form of) representation (4.11), or [26] where reductions of singular vectors were studied by other means)

$$
\text { (singular vector of } T_{\mathrm{m}} \text { minimal model) } \otimes(\text { ghost part) }
$$

Their BRST-primitives then become, upon factorization over the module generated by the null vector, the Lian-Zuckerman states in the theory of $c=-2$ matter dressed with gravity. The ghost number of a LZ state obtained in this way from an $\operatorname{MFF}$ vector $|\operatorname{MFF}\{r, s\}\rangle$ is equal to $r-j-1=\frac{1}{2}(r+1)-s$ for $r>j$ (where the ghost number of the $c=-2$ string counts the number of $C$ operators minus the number of $B$ operators and is zero for the $s l_{2}$-invariant ghost vacuum), and 0 for $r \leq j$. Such an accumulation of the LZ states in the ghost number 0 is related to the embedding pattern of the $c=28$ Virasoro Verma modules shown in (3.2) where, in the right column, every module is embedded into all the higher ones.

Moreover, the Verma module embedding diagrams then project as in (3.2), where the right column shows Virasoro Verma modules and dimensions of their ground states. Every Virasoro Verma module is embedded into all the higher ones. For the $s \ell(2)$ modules this is not so, instead there exist two $s \ell(2)$-modules (corresponding to two different spins $j$ ) that project onto the same Virasoro module (modules on the same level in (3.2)). Their embeddings add up to the 'complete' set of embeddings for the Virasoro Verma modules.

Our expression (3.9) for the $N=4$ singular vectors now gives rise, in view of (4.26), to an expression for singular vectors in the $c=28$ Virasoro Verma module. Rather curiously, writing these in a closed form requires the introduction of ghost systems (which, as noted above, decouple in the course of the evaluation of (3.9) in terms of the representation (4.25)). The possibility to arrive at such a representation for the Virasoro singular vectors rests on the fact that the representation (4.11) for the $s \ell(2)_{k=-4}$ currents (or, more generally, the $s \ell(2)_{k}$ representation from ref. [7]), unlike conventional 'bosonizations', does not imply the vanishing of any singular vector.

The explicit construction (3.9) does not, however, give all the singular vectors; as noted above, we have not continued it to all possible values of $r$ and $s$ in a closed form. This leaves aside a part of the $c=28$-singular vectors and the corresponding Lian-Zuckerman states. For these we only 
have explicit constructions in lower-level cases. In particular, the state $|\operatorname{MFF}\{1,2\}\rangle_{\mathcal{A}}$ gives rise to the ground ring generator $x$, and its explicit construction in terms of the $N=4$ generators reads

$$
x=B C b \Psi_{\mathrm{m}}-\frac{1}{2} \partial b \Psi_{\mathrm{m}}+\frac{1}{2} b \partial \Psi_{\mathrm{m}}=\left(\left(J_{N=4}^{0}\right)_{-1} \mathcal{G}_{0}^{2}+\frac{1}{2} \mathcal{G}_{-1}^{2}\right)\left(\overline{\mathcal{G}}^{1}\right)_{1}|1\rangle_{\mathcal{A}},
$$

where $\Psi_{\mathrm{m}}$ is the operator that corresponds to the state $|\Delta(1,2)\rangle$ in the matter sector. It would be interesting to obtain this operator in a systematic way, by extending the formula (3.9) along the lines of [30].

\section{$5 \quad N=4$ and matter + gravity}

In this section we will comment on the relation of the $N=4$ algebra represented in the cohomology of $\mathcal{Q}^{(0)}$ to the known symmetries of matter+gravity systems. The $N=4$ algebra (4.25) contains two twisted $N=2$ subalgebras, realized on a common energy-momentum tensor $\mathcal{T}$ and $U(1)$ current $\mathcal{H}=2 J^{0}$ :

$$
\left\{\begin{array} { r l } 
{ \mathcal { T } _ { 1 } } & { = \mathcal { T } } \\
{ \mathcal { H } _ { 1 } } & { = \mathcal { H } } \\
{ \mathcal { Q } _ { 1 } } & { = \mathcal { G } ^ { 1 } } \\
{ \mathcal { G } _ { 1 } } & { = \overline { \mathcal { G } } _ { 1 } }
\end{array} \quad \text { and } \quad \left\{\begin{array}{rl}
\mathcal{T}_{2} & =\mathcal{T} \\
\mathcal{H}_{2} & =\mathcal{H} \\
\mathcal{Q}_{2} & =\overline{\mathcal{G}}_{2} \\
\mathcal{G}_{2} & =\mathcal{G}^{2}
\end{array}\right.\right.
$$

In the first of these algebras, we can bosonize one of the ghost pairs as

$$
B=e^{i \varphi}, \quad C=e^{-i \varphi}
$$

and consider $\varphi$ as the Liouville scalar. Then the construction becomes the $k=-4$-case of the known $N=2$ representation [10] in terms of matter dressed with gravity:

$$
\begin{aligned}
\mathcal{Q}_{1} & =b \\
\mathcal{G}_{1} & =c\left(T-\frac{1}{2}(\partial \varphi)^{2}+\frac{\alpha_{+}+\alpha_{-}}{\sqrt{2}} \partial^{2} \varphi\right)+b c \partial c+\sqrt{2} \alpha_{+} \partial c \partial \varphi+\frac{1}{2}\left(1-2 \alpha_{+}^{2}\right) \partial^{2} c \\
\mathcal{H}_{1} & =-b c-\sqrt{2} \alpha_{+} \partial \varphi \\
\mathcal{T}_{1} & =T-\frac{1}{2}(\partial \varphi)^{2}+\frac{\alpha_{+}+\alpha_{-}}{\sqrt{2}} \partial^{2} \varphi-b \partial c
\end{aligned}
$$

where $\alpha_{-}=-\sqrt{k+2}, \alpha_{+}=-1 / \alpha_{-}$and, in our case, $k$ is set to $-4 . \quad T$ is the energy-momentum tensor of matter with central charge equal to $1-\frac{6(k+1)^{2}}{k+2}$ which becomes 28 when $k=-4$, in which case $T$ coincides with $T_{\mathrm{m}}$.

Similarly, the other twisted $N=2$ subalgebra from (5.1) becomes, after the bosonization

$$
b=e^{i \varphi}, \quad c=e^{-i \varphi}
$$

the other $N=2$ realization from 10 :

$$
\begin{aligned}
\mathcal{Q}_{2} & =C\left(T-\frac{1}{2}(\partial \varphi)^{2}+\frac{\alpha_{+}-\alpha_{-}}{\sqrt{2}} \partial^{2} \varphi\right)+B C \partial C+\sqrt{2} \alpha_{+} \partial C \partial \varphi+\frac{1}{2}\left(1-2 \alpha_{+}^{2}\right) \partial^{2} C \\
\mathcal{G}_{2} & =B \\
\mathcal{H}_{2} & =B C+\sqrt{2} \alpha_{+} \partial \varphi \\
\mathcal{T}_{2} & =T-\frac{1}{2}(\partial \varphi)^{2}+\frac{\alpha_{+}-\alpha_{-}}{\sqrt{2}} \partial^{2} \varphi-\partial B C-2 B \partial C
\end{aligned}
$$


evaluated at $k=-4$.

The two realizations (5.3) and (5.5) are related by an involutive automorphism of the twisted $N=2$ algebra [10]. Now we see that this automorphism lifts to the automorphism (2.27) of the $N=4$ algebra (4.25). It is induced, in the bosonized picture, by ghost permutations (cf. (2.28)

$$
b \leftrightarrow B, c \leftrightarrow C
$$

This acts as identity on the $s \ell(2)_{-4}$ algebra (4.11).

More generally, one can construct a 3-parameter family of $N=2$ algebras out of the $N=4$ generators (4.25) so that all members of this family share the energy-momentum tensor $\mathcal{T}$ and the $U(1)$ current $2 J_{N=4}^{0}$. These $N=2$ algebras are described by

$$
\begin{aligned}
\mathcal{T} & =T_{\mathrm{m}}-b \partial c-2 B \partial C-\partial B C, \\
\mathcal{H} & =b c-B C, \\
\mathcal{Q} & =a_{1} \overline{\mathcal{G}}_{2}+a_{2} \mathcal{G}^{1} \\
\mathcal{G} & =a_{3} \mathcal{G}^{2}+a_{4} \overline{\mathcal{G}}_{1}
\end{aligned}
$$

where $a_{1}, a_{2}, a_{3}, a_{4}$ are arbitrary parameters subject to the equation

$$
a_{1} a_{3}+a_{2} a_{4}=1
$$

Different algebras from the set (5.7) can be connected by a combination of transformations of the form $e^{-\oint \mathcal{A}}(\ldots) e^{\oint \mathcal{A}}$ with $\mathcal{A}$ being equal to:

$$
\begin{aligned}
& \mathcal{A}=p_{1} b c-p_{2} B C \\
& \text { or } \\
& \mathcal{A}=p\left(T_{\mathrm{m}} C c-C b \partial c c-B \partial C C c+C \partial^{2} c\right)
\end{aligned}
$$

On the set of parameters $a_{1}, a_{2}, a_{3}, a_{4}$ these transformations act as

$$
\begin{aligned}
& \left(a_{1}, a_{2}, a_{3}, a_{4}\right) \rightarrow\left(e^{p_{2}} a_{1}, e^{p_{1}} a_{2}, e^{-p_{2}} a_{3}, e^{-p_{1}} a_{4}\right) \\
& \text { and } \\
& \left(a_{1}, a_{2}, a_{3}, a_{4}\right) \rightarrow\left(a_{1}+p a_{2}, a_{2}, a_{3}, a_{4}+p a_{3}\right)
\end{aligned}
$$

respectively. As one can see, not every two algebras of the family (5.7) can be mapped into each other by such a transformation. The space of parameters $a_{1}, a_{2}, a_{3}, a_{4}$ falls into three domains:

$$
\begin{gathered}
\left(a_{1}, 0, a_{3}, a_{4}\right) \\
\left(a_{1}, a_{2}, 0, a_{4}\right) \\
\left(a_{1}, a_{2}, a_{3}, a_{4}\right)
\end{gathered}
$$

where in the latter case neither $a_{2}$ nor $a_{3}$ is equal to zero. For an algebra that belongs to the first domain (that is $a_{2}=0$, hence $a_{3} \neq 0$ from (5.8)) the cohomology of $\mathcal{G}$ is trivial, because a dimension-(-1) field exists:

$$
\Psi_{1}^{\mathcal{G}}=\frac{1}{a_{3}} C-\frac{a_{4}}{a_{3}^{2}} c \partial C C
$$


that is conjugate to $\mathcal{G}$ :

$$
\mathcal{G}(z) \Psi_{1}^{\mathcal{G}}(w)=\frac{1}{z-w}
$$

In the case of domain 2, we have a reversed situation. A dimension-0 field:

$$
\Psi_{2}^{\mathcal{Q}}=\frac{1}{a_{2}} c-\frac{a_{1}}{a_{2}^{2}} C \partial c c, \quad a_{2} \neq 0
$$

is conjugate to $\mathcal{Q}$, while the one conjugate to $\mathcal{G}$ does not exist. In this case the cohomology of $\mathcal{Q}$ are trivial. In the last case (with neither $a_{2}$ nor $a_{3}$ being zero) we have that $\Psi_{3}^{\mathcal{Q}}=\Psi_{2}^{\mathcal{Q}}$ as well as $\Psi_{3}^{\mathcal{G}}=\Psi_{1}^{\mathcal{G}}$ exist. Hence the cohomology of both $\mathcal{Q}$ and $\mathcal{G}$ are trivial. Therefore the would-be transformations connecting different domains do not exist, since such transformations are required to preserve the OPEs. The set of transformations of the form $e^{-\oint \mathcal{A}}(\ldots) e^{\oint \mathcal{A}}$ act transitively on each domain. The above algebras (5.3) and (5.5) correspond, obviously, to $(1,0,1,0)$ and $(0,1,0,1)$. At the same time, the $N=2$ algebra (2.21), restricted to the cohomology of $\mathcal{Q}^{(0)}$ in the bosonized description, is identified as the $\left(1,1, \frac{1}{2}, \frac{1}{2}\right)$ algebra.

The appearance of field operators such as the above $\Psi$ is often characteristic to bosonized pictures; thus, for example, the cohomology of $\mathcal{Q}_{\mathcal{A}}$ is trivialized in the bosonized picture, since there exists a dimension-0 field

$$
\Psi=c-C \partial c c+C c \mathcal{B}^{0}
$$

that is conjugate to the BRST current $\mathcal{J}_{\mathcal{A}}$ :

$$
\mathcal{J}_{\mathcal{A}}(z) \Psi(w)=\frac{1}{z-w}
$$

whence $\left\{\mathcal{Q}_{\mathcal{A}}, \Psi_{0}\right\}=1$ and we conclude that every $\mathcal{Q}_{\mathcal{A}}$ closed state is $\mathcal{Q}_{\mathcal{A}}$ exact. This vanishing of the cohomology is an example of the Koszul trivialization, described e.g. in [31]. It occurs when extending the algebra $\mathcal{A}(2.2)$ to an algebra $\overline{\mathcal{A}}$ and extending the BRST operator appropriately, in such a way that any $\mathcal{Q}_{\mathcal{A}}$-closed state is given by $\mathcal{Q}_{\mathcal{A}}$ acting on one of the 'new' states. The appearance of the above $\Psi$ is due to explicitly solving the condition $\widehat{J}^{0} \sim 0$. Indeed, taking $\widehat{J}^{0}$ to be an 'elementary' field and parametrizing the fields orthogonal to $\widehat{J}^{0}$ in terms of other 'elementary' fields, one has to introduce, one way or another, $\left(J^{+}\right)^{-1}$ (in our realization, this was simply $e^{-\bar{\phi}}$ ). Allowing $\left(J^{+}\right)^{-1}$ to appear leads to the existence of an operator such as the above $\Psi$ that trivializes the cohomology. To restore the 'original' cohomology of $\mathcal{Q}_{\mathcal{A}}$, one has to project from the bosonized algebra $\overline{\mathcal{A}}$ to $\mathcal{A}$ itself. Recall that, generally, the cohomology of $\mathcal{Q}_{\mathcal{A}}$ can be evaluated using the spectral sequence associated with the decomposition (2.19). While the cohomology of $\mathcal{Q}^{(0)}$ and $\mathcal{Q}^{(1)}=\mathcal{Q}_{\text {str }}$ do not necessarily vanish, it is the cohomology of $\mathcal{Q}^{(2)}=\oint \mathcal{G}^{1}$ that undergoes the Koszul trivialization due to the appearance of the $c$ field in the bosonized picture. Note, however, that the $c$ ghost is not a part of the $N=4$ algebra, and thus it is possible to keep the non-trivial cohomology by working solely in the representation of the $N=4$ algebra.

\section{Concluding remarks}

We have presented arguments showing that the $k=-4 s \ell(2)$ WZW model is cohomologically equivalent to the bosonic string with $c=-2$ matter. We have observed the presence of an $N=4$ symmetry in this system, which points to an $N=4$ origin of the Lian-Zuckerman states in the $c=-2$ bosonic string. The derivation involves a spectral sequence on the $s \ell(2)_{-4}$ BRST complex and can 
be viewed as an extension of the Universal string ideology to include theories with Kač-Moody symmetries 6 . While the $N=4$ algebra is specific to $c=-2$ matter, the relation of Lian-Zuckerman states to the $s \ell(2)_{k}$ algebra is likely to hold in general, since a twisted $N=2$ is always present in non-critical strings, and on the other hand the relevant $N=2$ singular vectors are isomorphic with $s \ell(2)$ singular vectors [30]. The appearance of the $N=4$ algebra would also be interesting to understand in terms of geometry of flag manifolds, along the lines of ref. [28].

Acknowledgements. We are grateful to B. Feigin for useful discussions. We also thank O. Andreev, J.M. Figueora-O'Farrill, S. Hwang, O. Khudaverdyan, A. Marshakov, A. Taormina, I.V. Tyutin, M.A. Vasiliev, and B.L. Voronov. The research described in this publication was made possible in part by Grant \#MQM300 from the International Science Foundation and Government of Russian Federation, and by RFFI grant 94-02-06338-a.

\section{References}

[1] O. Aharony, O. Ganor, J. Sonnenschein, S. Yankielowicz, and N. Sochen, Nucl. Phys. B399 (1993) 527;

O. Aharony, J. Sonnenschein, and S. Yankielowicz, Phys. Lett. B289 (1992) 309;

O. Aharony, O. Ganor, J. Sonnenschein, and S. Yankielowicz, Nucl. Phys. B399 (1993) 560.

[2] H.-L. Hu and M. Yu, Nucl. Phys. B391 (1993) 389.

[3] P. Bouwknegt, J. McCarthy, and K. Pilch Semi-infinite cohomology in conformal field theory, and 2d gravity, CERN-TH.6646/92.

[4] S. Hwang and H. Rhedin, Construction of BRST invariant states in $G / H$ WZNW models, Göteborg ITP 94-26 (December 1994).

[5] F.G. Malikov, B.L. Feigin, and D.B. Fuchs, Funk. Anal. Prilozh. 20 no 2 (1987) 25.

[6] B.H. Lian and G.J. Zuckerman, Phys. Lett. B254 (1991) 417.

[7] A.M. Semikhatov, Mod. Phys. Lett. A9 (1994) 1867.

[8] J. Distler Nucl. Phys. B342 (1990) 523.

[9] S. Mukhi and C. Vafa, Nucl. Phys. B407 (1993) 667;

S. Mukhi, The Two-dimensional string as a topological field theory, TIFR-TH-93-61.

[10] B. Gato-Rivera and A.M. Semikhatov, Nucl. Phys. B408 (1993) 133.

11] M. Bershadsky, W. Lerche, D. Nemeschansky, and N.P. Warner, Nucl. Phys. B401 (1993) 304.

[12] N. Berkovits and C. Vafa, Mod. Phys. Lett. A9 (1994) 653.

[13] J.M. Figueora-O'Farrill, Phys. Lett. B321 (1994) 344.

14] H. Ishikawa and M. Kato, Mod. Phys. Lett. A9 (1994) 725.

[15] D. Karabali and H. Schnitzer, Nucl. Phys. B329 (1990) 649.

[16] J.M. Isidro and A.V. Ramallo Phys. Lett. B316 (1993) 488.

[17] B. Feigin and E. Frenkel, Int. J. Mod. Phys. A7, Suppl. 1A (1992) 197.

[18] D.H. Friedan, E.J. Martinec, and S.H. Shenker, Nucl. Phys. B271 (1986) 93.

19] Y. Kazama, Mod. Phys. Lett. A6, 14, 1321 (1991).

[20] T. Eguchi and S.-K. Yang, Mod. Phys. Lett. A4 (1990) 1653.

[21] E. Witten, Commun. Math. Phys. 118 (1988) 411; Nucl. Phys. B340 (1990) 281.

[22] S. Matsuda, KUCP-42 (December 1991).

[23] T. Eguchi and A. Taormina, Phys. Lett. B196 (1986) 75.

[24] J.L. Petersen and A. Taormina, Coset construction and character sumrules for the doubly extended $N=4$ superconformal algebras, DTP/92/49, NBI-HE-92-73 (November 1992).

[25] M. Bershadsky and H. Ooguri, Commun. Math. Phys. 126 (1989) 49.

[26] A.Ch. Ganchev and V.B. Petkova, Phys. Lett. B318 (1993) 77; Phys. Lett. B293 (1992) 56; P. Furlan, A.Ch. Ganchev, R. Paunov and V.B. Petkova, Nucl. Phys. B394 (1993) 665.

\footnotetext{
${ }^{6}$ With the help of the explicit realization for the $s \ell(2)$ currents (in terms of matter, Liouville and ghosts) one can also construct a homotopy transformation relating the $s \ell(2)$ space of states in the chosen realization with states of the matter+gravity theories.
} 
[27] M. Bauer and N. Sochen, Fusion and singular vectors in $A_{1}^{(1)}$ highest weight cyclic modules, hep-th@xxx/9201079.

[28] B.L. Feigin and A.V. Stoyanovsky, Quasi-particle models for the representations of Lie algebras and geometry of flag manifold, RIMS-942 (Sept. 1993).

[29] A. Schwimmer and N. Seiberg, Phys. Lett. B184 (1987) 191.

[30] A.M. Semikhatov and I.Yu. Tipunin, Singular Vectors of the Topological Conformal Algebra, hep-th@xxx/9512079.

[31] F. Akman, Some cohomology operators an 2-D field theory, MSRI preprint, and PhD thesis, Yale. 